\title{
Forbidden Configurations and Product Constructions
}

\author{
R.P. Anstee*1, Christina Koch ${ }^{\dagger 1}$, Miguel Raggi ${ }^{\ddagger 1}$, A. Sali ${ }^{\S 2}$ \\ 1 Mathematics Department, The University of British Columbia, Vancouver, B.C. Canada V6T \\ $1 \mathrm{Z2}$ \\ 2 Alfréd Rényi Institute, Budapest, Hungary
}

\begin{abstract}
A simple matrix is a $(0,1)$-matrix with no repeated columns. For a $(0,1)$-matrix $F$, we define that a $(0,1)$-matrix $A$ has $F$ as a configuration if there is a submatrix of $A$ which is a row and column permutation of $F$ (trace is the set system version of a configuration). Let $\|A\|$ denote the number of columns of $A$. We define

forb $(m, F)=\max \{\|A\|: A$ is $m$-rowed simple matrix and has no configuration $F\}$.

We extend this to a family $\mathcal{F}=\left\{F_{1}, F_{2}, \ldots, F_{t}\right\}$ and define

forb $(m, \mathcal{F})=\max \{\|A\|: A$ is $m$-rowed simple matrix and has no configuration $F \in \mathcal{F}\}$.

We consider products of matrices. Given an $m_{1} \times n_{1}$ matrix $A$ and an $m_{2} \times n_{2}$ matrix $B$, we define the product $A \times B$ as the $\left(m_{1}+m_{2}\right) \times n_{1} n_{2}$ matrix columns consist of all possible combinations obtained from placing a column of $A$ on top of a column of $B$. Let $I_{k}$ denote the $k \times k$ identity matrix, let $I_{k}^{c}$ denote the $(0,1)$-complement of $I_{k}$ and let $T_{k}$ denote the $k \times k$ upper triangular $(0,1)$-matrix with a 1 in position $i, j$ if and only if $i \leq j$. We show forb $\left(m,\left\{I_{2} \times I_{2}, T_{2} \times T_{2}\right\}\right)$ is $\Theta\left(\mathrm{m}^{3 / 2}\right)$ while obtaining a linear bound when forbidding all 2-fold products of all $2 \times 2(0,1)$-simple matrices. For two matrices $F, P$, where $P$ is $m$-rowed, let $f(F, P)=\max _{A}\{\|A\|: A$ is $m$-rowed submatrix of $P$ with no configuration $F\}$. We establish $f\left(I_{2} \times I_{2}, I_{m / 2} \times I_{m / 2}\right)$ is $\Theta\left(m^{3 / 2}\right)$ whereas $f\left(I_{2} \times T_{2}, I_{m / 2} \times T_{m / 2}\right)$ and $f\left(T_{2} \times T_{2}, T_{m / 2} \times T_{m / 2}\right)$ are both $\Theta(m)$. Additional results are obtained. We use the results on patterns due to Marcus and Tardos and generalizations due to Klazar and Marcus, Balogh, Bollobás and Morris.
\end{abstract}

Key words. VC-dimension, forbidden configurations, trace, patterns, products

\section{Introduction}

The investigations into the extremal problem of the maximum number of edges in an $n$ vertex graph with no subgraph $H$ originated with Erdős and Stone [11] and Erdös and Simonovits [10]. There is a large and illustrious literature. There are several ways to generalize to the hypergraph setting. Typically we consider simple hypergraphs, namely those with no repeated edges. One can consider a $r$-uniform hypergaph $H$ and forbid a given subhypergraph $H^{\prime}$, itself a $r$-uniform hypergraph. One can consider a $r$-uniform

\footnotetext{
* Research supported in part by NSERC and Hungarian National Research Fund (OTKA) grant no. NK 78439

$\dagger$ Research supported in part by NSERC of first author

$¥$ Research supported in part by NSERC of first author

$\S$ Research was supported in part by Hungarian National Research Fund (OTKA) grant no. NK 78439
} 
hypergraph $H$ and forbid a given trace. Or one can extend to general hypergraphs and forbid a given trace. This latter problem in the language of matrices is our focus. We say a matrix is simple if it is a $(0,1)$-matrix and there are no repeated columns. Given a $(0,1)$-matrix $F$, we say a matrix $A$ has $F$ as a configuration denoted $F \in A$, if there is a submatrix of $A$ which is a row and column permutation of $F$. Let $\|A\|$ denote the number of columns in $A$. We define

forb $(m, F)=\max \{\|A\|: A$ is $m$-rowed simple matrix with no configuration $F\}$. We recall an important conjecture from [5]. Let $I_{k}$ denote the $k \times k$ identity matrix, $I_{k}^{c}$ denotes the $(0,1)$-complement of $I_{k}$, and $T_{k}$ denotes the $k \times k$ upper triangular matrix whose $i$ th column has 1 's in rows $1,2, \ldots, i$ and 0 's in the remaining rows. For $p$ matrices $m_{1} \times n_{1}$ matrix $A_{1}$, an $m_{2} \times n_{2}$ matrix $A_{2}, \ldots$, an $m_{p} \times n_{p}$ matrix $A_{p}$ we define $A_{1} \times A_{2} \times$ $\cdots \times A_{p}$ as the $\left(m_{1}+\cdots+m_{p}\right) \times n_{1} n_{2} \cdots n_{p}$ matrix whose columns consist of all possible combinations obtained from placing a column of $A_{1}$ on top of a column of $A_{2}$ on top of a column of $A_{3}$ etc. For example the vertex-edge incidence matrix of the complete bipartite graph $K_{m / 2, m / 2}$ is $I_{m / 2} \times I_{m / 2}$. Define $\mathbf{1}_{k}$ to be the $k \times 1$ column of 1 's and $\mathbf{0}_{\ell}$ to be the $\ell \times 1$ columns of 0 's. We can define $\mathbf{1}_{k} \mathbf{0}_{\ell}$ to be the $(k+\ell) \times 1$ column $\mathbf{1}_{k} \times \mathbf{0}_{\ell}$.

Conjecture 1. [5] Let $F$ be a $k \times \ell$ matrix with $F \neq \mathbf{1}_{1} \mathbf{0}_{1}$. Let $X(F)$ denote the largest $p$ such that there are choices $A_{1}, A_{2}, \ldots, A_{p} \in\left\{I_{m / p}, I_{m / p}^{c}, T_{m / p}\right\}$ so that $F \notin A_{1} \times A_{2} \times$ $\cdots \times A_{p}$, then forb $(m, F)=\Theta\left(m^{X(F)}\right)$.

We are assuming $p$ divides $m$ which does not affect asymptotic bounds. We obtain evidence that supports the conjecture while also indicating some potential difficulties. We will be considering $F$ that are products of 2 -rowed matrices. The following are the maximal 2-rowed simple submatrices of the matrices $I, T, I^{c}$ of the conjecture. Let

$$
E_{1}=\left[\begin{array}{lll}
0 & 1 & 0 \\
0 & 0 & 1
\end{array}\right], \quad E_{2}=\left[\begin{array}{lll}
0 & 1 & 1 \\
0 & 0 & 1
\end{array}\right], \quad E_{3}=\left[\begin{array}{lll}
1 & 0 & 1 \\
0 & 1 & 1
\end{array}\right]
$$

For an $m$-rowed matrix $P$, we define

$$
f(F, P)=\max \{\|A\|: A \text { is } m \text {-rowed submatrix of } P \text { with no configuration } F\} .
$$

Let $K_{m}$ denote the $m \times 2^{m}$ simple matrix consisting of all possible different columns. Then $\operatorname{forb}(m, F)=f\left(F, K_{m}\right)$.

Theorem 1. $f\left(E_{1} \times E_{1}, I_{m / 2} \times I_{m / 2}\right)$ is $\Theta\left(m^{3 / 2}\right)$.

Theorem 2. $f\left(E_{1} \times E_{2}, I_{m / 2} \times T_{m / 2}\right) \leq 2 m$.

Theorem 3. $f\left(E_{2} \times E_{2}, T_{m / 2} \times T_{m / 2}\right) \leq 2 m$.

The bound of Theorem 1 is perhaps unexpected in view of Conjecture 1 but it is not a counterexample. The remaining three cases $\left(E_{1} \times E_{3}\right.$ in $I_{m / 2} \times I_{m / 2}^{c}, E_{2} \times E_{3}$ in $T_{m / 2} \times I_{m / 2}^{c}$ and $E_{3} \times E_{3}$ in $\left.I_{m / 2}^{c} \times I_{m / 2}^{c}\right)$ follow by taking appropriate $(0,1)$-complements. The proof of Theorem 3 is in Section 3, the proof of Theorem 1 is in Section 4 and the proof of Theorem 2 is in Section 5. Related results such as $f\left(E_{1} \times E_{2} \times E_{2}, I_{m / 3} \times T_{m / 3} \times T_{m / 3}\right)$ is $\Theta\left(m^{2}\right)$ being $\Theta\left(m^{2}\right)$ (Lemma 6) are proved in Section 3, Section 4, Section 5.

A central idea to many of our proofs is to encode columns of a $p$-fold product $A_{1} \times$ $A_{2} \times \cdots \times A_{p}$ as 1 's in a $p$ dimensional $(0,1)$-array whose $i$ th coordinate is indexed by 
the columns of $A_{i}$. In Section 2 we use results about patterns including the fundamental result of Marcus and Tardos [16]) and generalizations of Klazar and Marcus [14], Balogh, Bollobás and Morris [8]. We establish some exact bounds for some small cases. We relate patterns to forbidden configuration results in Section 3. The following basic result is proven in Section 3.

Proposition 1. Let $p, q, r, u, v, w$ be given positive integers. Define $x^{+}=\max \{0, x\}$. The configuration given by the product $F=\overbrace{E_{1} \times \cdots \times E_{1}}^{u} \times \overbrace{E_{2} \times \cdots \times E_{2}}^{v} \times \overbrace{E_{3} \times \cdots \times E_{3}}^{w}$ is contained in the a-fold product $\overbrace{I_{m / a} \times \cdots \times I_{m / a}}^{p} \times \overbrace{T_{m / a} \times \cdots \times T_{m / a}}^{p} \times \overbrace{I_{m / a}^{c} \times \cdots \times I_{m / a}^{c}}^{q}$ (where we have set $a=p+q+r$ ) if and only if

$$
2\left((u-p)^{+}+(v-q)^{+}+(w-r)^{+}\right) \leq(p-u)^{+}+(q-v)^{+}+(r-w)^{+} .
$$

For example with $u=2, q=3$ and the rest being 0 , Proposition 1 yields that $E_{1} \times E_{1} \notin$ $T \times T \times T$ and hence $\operatorname{forb}\left(m, E_{1} \times E_{1}\right)$ is $\Omega\left(m^{3}\right)$.

We now consider forbidden families of configurations. We have noted (in [3]) that forb $\left(m,\left\{E_{1}, E_{2}, E_{3}\right\}\right)=2$. Balogh and Bollobás [7] have the much more general result that for a given $k$, there is a constant $c_{k}$ such that forb $\left(m,\left\{I_{k}, T_{k}, I_{k}^{c}\right\}\right)=c_{k}$.

Let $\left\{E_{1}, E_{2}, E_{3}\right\} \times\left\{E_{1}, E_{2}, E_{3}\right\}$ denote the 6 possible 2-fold products whose terms are chosen from $\left\{E_{1}, E_{2}, E_{3}\right\}$. We would like to compute forb $\left(m,\left\{E_{1}, E_{2}, E_{3}\right\} \times\left\{E_{1}, E_{2}, E_{3}\right\}\right)$ but in the interest of a more tractable proof we consider $I_{2}$ as a replacement for both $E_{1}$ and $E_{3}\left(I_{2}^{c}\right.$ is the same configuration as $\left.I_{2}\right)$ and $T_{2}$ as replacement for $E_{2}$. We note forb $\left(m,\left\{I_{2}, T_{2}\right\}\right)=2$ and the bounds of Theorem 1, Theorem 2 and Theorem 3 apply. In Section 6 we prove:

Theorem 4. forb $\left(m,\left\{I_{2} \times I_{2}, T_{2} \times T_{2}\right\}\right)$ is $\Theta\left(m^{3 / 2}\right)$.

$$
I_{2} \times I_{2}=\left[\begin{array}{llll}
1 & 1 & 0 & 0 \\
0 & 0 & 1 & 1 \\
1 & 0 & 1 & 0 \\
0 & 1 & 0 & 1
\end{array}\right], T_{2} \times T_{2}=\left[\begin{array}{llll}
1 & 1 & 1 & 1 \\
0 & 0 & 1 & 1 \\
1 & 1 & 1 & 1 \\
0 & 1 & 0 & 1
\end{array}\right] .
$$

We make novel use of our standard decomposition (4) that has been useful studying forbidden configurations in the past. We also prove the following exact bound in Section 7 , which contrasts with Theorem 4 . The following four matrices are all $2 \times 2$ simple matrices (up to row and column permutations). Let

$$
I_{2}=\left[\begin{array}{ll}
1 & 0 \\
0 & 1
\end{array}\right] \quad T_{2}=\left[\begin{array}{ll}
1 & 1 \\
0 & 1
\end{array}\right] \quad U_{2}=\left[\begin{array}{ll}
0 & 0 \\
0 & 1
\end{array}\right] \quad V_{2}=\left[\begin{array}{ll}
1 & 0 \\
1 & 0
\end{array}\right] .
$$

We note forb $\left(m,\left\{I_{2}, T_{2}, U_{2}, V_{2}\right\}\right)=1$. Define $\quad\left\{I_{2}, T_{2}, U_{2}, V_{2}\right\} \times\left\{I_{2}, T_{2}, U_{2}, V_{2}\right\}$ $=\left\{X \times Y: X, Y \in\left\{I_{2}, T_{2}, U_{2}, V_{2}\right\}\right\}$ as the 10 possible products of these matrices.

Theorem 5. We have forb $\left(m,\left\{I_{2}, T_{2}, U_{2}, V_{2}\right\} \times\left\{I_{2}, T_{2}, U_{2}, V_{2}\right\}\right)=m+3$.

The following definitions are used. Let $[n]=\{1,2, \ldots, n\}$. We discuss $d$-dimensional $(0,1)$-arrays $B$. We define $\sigma_{1}(B)$ to be the number of 1 's in $B$. Thinking of the positions in $B$ as elements of $[n]^{d}$, we let the coordinates of $B$ be $x_{1}, x_{2}, \ldots, x_{d}$ and for a position $\mathbf{y} \in[n]^{d}$ we define $x_{i}(\mathbf{y})$ to be the value of coordinate $x_{i}$ in $\mathbf{y}$. Let $\operatorname{proj}_{i}(B)$ denote the 
$[n]^{d-1}(d-1)$-dimensional $(0,1)$-array obtained from $B$ by projecting in the direction $e_{i}$ (where $e_{i}$ is the $d$-dimensional $(0,1)$-vector with a single 1 in coordinate $x_{i}$ ). For each position $\mathbf{y} \in[n]^{d}$ we form $\mathbf{y}_{\bar{i}}$ in $[n]^{d-1}$ by deleting the $i$ th coordinate of $\mathbf{y}$. If $B$ has a 1 in position $\mathbf{z}$, then we place a 1 in position $\mathbf{z}_{\bar{i}}$ in $\operatorname{proj}_{i}(B)$. We repeat for all entries of $B$. All other entries in $\operatorname{proj}_{i}(B)$ are 0 . For example if $B$ is a $2 \times 2 \times 23$-dimensional array with 1 's in positions $(1,1,2),(1,2,1),(2,2,1)$ and then $\operatorname{proj}_{1}(B)$ has two 1 's in positions $(1,2),(2,1)$ while $\operatorname{proj}_{i} 2(B)$ has three 1's in positions $(1,2),(1,1),(2,1)$.

\section{Splits}

We are going to consider the maximum number of 1 's in a $n_{1} \times n_{2}(0,1)$-matrix $A$ subject to some property. The problems in this section are close relatives of Zarankiewicz' problem $[15],[12]$ and indeed the investigations of patterns $[13],[16],[17],[14]$ and have the slightly geometric flavour of points in space.

For any subset $R(1) \subset\left[n_{1}\right]$ and $R(2) \subset\left[n_{2}\right]$ we define $\left.A\right|_{(R(1), R(2))}$ as the submatrix of $A$ formed of the entries contained in the rows $R(1)$ and in the columns $R(2)$. In this section we will be considering cases where both $R(1)$ and $R(2)$ consist of consecutive integers. Let $p_{1}, p_{2}$ be given with $1 \leq p_{j} \leq n_{j}$ for $j=1,2$. Assume we are given $I(j)=$ $\left\{r_{1}(j), r_{2}(j), \ldots, r_{p-1}(j)\right\}$ with $0<r_{1}(j)<r_{2}(j)<\cdots<r_{p_{j}-1}(j)<n_{j}$ for $j=1,2$. Define $R_{1}(j)=\left\{1,2, \ldots, r_{1}(j)\right\}, R_{i}(j)=\left\{r_{i-1}(j)+1, r_{i-1}(j)+2, \ldots, r_{i}(j)\right\}$ for $1<i<p_{j}$ and $R_{p_{j}}(j)=\left\{r_{p_{j}-1}(j)+1, r_{p_{j}-1}(j)+2, \ldots, n_{j}\right\}$. We observe that $\cup_{i=1}^{p_{j}} R_{i}(j)=\left[n_{j}\right]$. We say that $A$ has a $p_{1}, p_{2}$ split if there are choices $I(1), I(2)$ and hence $R_{i}(1)$ for $1 \leq i \leq p_{1}$ and $R_{j}(2)$ for $1 \leq j \leq p_{2}$ so that $\left.A\right|_{\left(R_{i}(1), R_{j}(2)\right)}$ is a non zero matrix for all choices $1 \leq i \leq p_{1}$ and $1 \leq j \leq p_{2}$. Let $g(m, n ; k, k)$ denote the maximum number of 1 's in a $m \times n(0,1)$ matrix that does not have a $k, k$ split. Below is an example of a 3,3 split where a 1 from each block is indicated

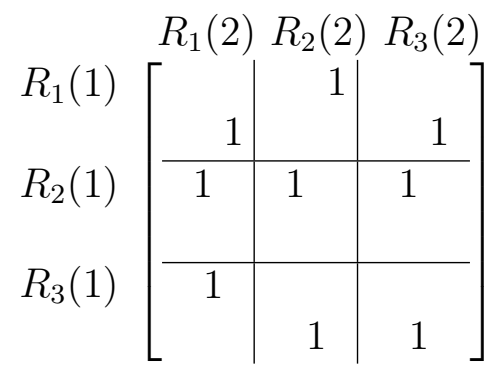

\section{A 3,3 split}

Theorem 6. Marcus and Tardos [16]. Let $k$ be given. Then there exists a constant $c_{k}$ such that $g(n, n ; k, k) \leq c_{k} n$.

The result in [16] involving forbidden permutation patterns implies the above result by choosing the permutation appropriately. Moreover the proof directly extends to the above result. While the constants involved in [16] are not optimal, we can produce best possible constants for small values:

Theorem 7. Let $m, n$ be given with $m, n \geq 2$. Then $g(m, n ; 2,2)=m+n-1$ and $g(m, n ; 3,3)=2 m+2 n-4$.

Proof: An $m \times n$ matrix $B$ with $\left(p_{1}-1\right) m+\left(p_{2}-1\right) n-\left(p_{1}-1\right)\left(p_{2}-1\right) 1$ 's can be constructed with 1 's in the first $p_{1}-1$ rows and the first $p_{2}-1$ columns. Then $B$ has no $p_{1}, p_{2}$ split. 
The following graph theory argument proves the upper bound for $p_{1}=2$ and $p_{2}=2$. Consider the bipartite graph on $m+n$ vertices $r_{1}, r_{2}, \ldots, r_{m}, c_{1}, c_{2}, \ldots, c_{n}$ given by the matrix $A$ where a 1 in entry $i, j$ joins vertex $r_{i}$ to $c_{j}$. If $\sigma_{1}(A) \geq m+n$, then the bipartite graph has a cycle. If we consider the smallest column index $c_{1}$ of a vertex of the cycle which joins two vertices $r_{1}<r_{2}$, then setting $I(1)=\left\{r_{1}\right\}$ and $I(2)=\left\{c_{1}\right\}$ yield a 2,2 split of $A$.

Now consider $p_{1}=p_{2}=3$. Let $A$ be a given $m \times n(0,1)$-matrix with $\sigma_{1}(A)>2 m+2 n-4$. Now create a new matrix $A^{\prime}$ from $A$ by deleting, if possible, the topmost 1 and bottommost 1 in each column and so $\sigma_{1}\left(A^{\prime}\right) \geq \sigma_{1}(A)-2 n$. Note that we use deleting a 1 to refer to replacing a 1 by a 0 . Now create a new matrix $A^{\prime \prime}$ from $A^{\prime}$ by deleting, if possible, the two remaining rightmost 1 's in each row and so $\sigma_{1}\left(A^{\prime \prime}\right) \geq \sigma_{1}\left(A^{\prime}\right)-(2 m-4)$ where we note that $A^{\prime}$ has no $1^{\prime}$ 's in the first or last row. By hypothesis, $\sigma_{1}\left(A^{\prime \prime}\right)>0$. Say there is a 1 in $A^{\prime \prime}$ in position $r_{2}, c_{1}$. By construction there are two entries in $A^{\prime}$ to the right of that 1 , say in positions $r_{2}, c_{2}$ and $r_{2}, c_{3}$. By construction for each of these three entries there are 1 's above and below in $A$ in columns $c_{1}, c_{2}, c_{3}$. We now identify a 3,3 split in $A$ by setting $I(1)=\left\{r_{2}-1, r_{2}\right\}$ and $I(2)=\left\{c_{1}, c_{2}\right\}$. We conclude that $g(m, n ; 3,3) \leq 2 m+2 n-4$.

This proof technique was introduced to the authors by Jozsef Solymosi as a curling technique (the winter sport of curling uses a strategy called 'peeling').

The papers [14],[8] consider Theorem 6 generalized to $d$-dimensional arrays. The following is our notation. Given integers $n_{1}, n_{2}, \ldots, n_{d}$ we can consider the positions $\prod_{i=1}^{d}\left[n_{i}\right]$ in an $n_{1} \times n_{2} \times \cdots \times n_{d}(0,1)$-array $A$. Our main interest is in the case $n_{1}=n_{2}=$ $\cdots=n_{d}$. Let $p_{1}, p_{2}, \ldots, p_{d} \geq 2$ be given. Assume we have $d$ sets of indices $I(j)=$ $\left\{r_{1}(j), r_{2}(j), \ldots, r_{p_{j}-1}(j)\right\}$ for coordinate $j$, for $j=1,2, \ldots, d$. We can form $d$ sets $R_{1}(j), R_{2}(j), \ldots, R_{p_{j}}(j)$ with $\cup_{i=1}^{p_{j}} R_{i}(j)=\left[n_{i}\right]$ as follows: $R_{1}(j)=\left\{1,2, \ldots, r_{1}(j)\right\}, R_{2}(j)=$ $\left\{r_{1}(j)+1, r_{1}(j)+2, \ldots, r_{2}(j)\right\}, \ldots, R_{p_{j}}(j)=\left\{r_{p_{j}-1}(j)+1, r_{p_{j}-1}(j)+2, \ldots, n_{j}\right\}$. We say $A$ has a $p_{1}, p_{2}, \ldots, p_{d}$ split if we can choose the sets as above and for each $j \in[d]$ and for each possible choice $t \in\left[p_{j}\right]$ with $R(j)=R_{t}(j)$, the $\prod_{i=1}^{d} p_{j}$ block $\left.A\right|_{(R(1), R(2), \ldots, R(d))}$ contains at least one 1 . Let $g\left(n_{1}, n_{2}, \ldots, n_{d} ; p_{1}, p_{2}, \ldots, p_{d}\right)$ be the maximum number of 1 's in $n_{1} \times n_{2} \times \cdots \times n_{d}(0,1)$-array that has no $p_{1}, p_{2}, \ldots, p_{d}$ split. The following yields the asymptotics.

Theorem 8. Klazar and Marcus [14], Balogh, Bollobás and Morris [8]. Let k, $d$ be given. Then there exists a constant $c_{k, d}$ so that $g(\overbrace{n, \ldots, n}^{d} ; \overbrace{k, \ldots, k}^{d}) \leq c_{k, d} n^{d-1}$.

The easy way to have many 1's in a $d$-dimensional array and have no $p_{1}, p_{2}, \ldots, p_{d}$ split is to place 1's in all positions which, for some choice $i \in[d]$ has the $i$ th coordinate less than $p_{i}$. There will be $\prod_{i=1}^{d} p_{i}$ blocks and the block $\left(R_{p_{1}}(1), R_{p_{2}}(2), \ldots, R_{p_{d}}(d)\right)$ will have a 1 and its coordinate in the $i$ th direction will be at least $p_{i}$. In general this is not optimal.

We may extend the argument in Theorem 7 to $\overbrace{3,3, \ldots, 3}^{d-1}, q$ splits of $d$-dimensional arrays. It is surprising that we get exact results here yet do not have a reasonable bound for $g(m, n ; 4,4)$ but can conjecture $g(m, m ; 4,4)=7 m-13$.

Theorem 9. Let $B$ be the $\prod_{i=1}^{d}\left[m_{i}\right](0,1)$-array with 1 's in entries whose $j$ coordinate is 1 or 2 for some $j=1,2, \ldots, d-1$ or whose $d$ th coordinate is $1,2, \ldots$ or $q-1$. The matrix 
$B$ has no $\overbrace{3,3, \ldots, 3}^{d-1}, q$ split. Let $A$ be any $\prod_{i=1}^{d}\left[m_{i}\right](0,1)$-array with no $\overbrace{3,3, \ldots, 3}^{d-1}, q$ split. Then $\sigma_{1}(A) \leq \sigma_{1}(B)$, and hence for $q=3, g(m_{1}, m_{2}, \ldots, m_{d} ; \overbrace{3,3, \ldots, 3}^{d-1}, q)=\sigma_{1}(B)$.

Proof: Let $A$ be any $\prod_{i=1}^{d}\left[m_{i}\right](0,1)$-array with no $\overbrace{3,3, \ldots, 3}^{d-1}, q$ split. For each direction $e_{i}$ with $i=1,2, \ldots, d-1$, delete from $A$, if possible, the two 1 's with the smallest and largest coordinate value $x_{i}$. Finally in the direction $e_{d}$, delete from $A$, if possible, the largest $q-1$ points in each line in the direction $e_{d}$. Let $A^{\prime}$ be the resulting $(0,1)$-array.

Now if $A^{\prime}$ has a 1 in position $\left(y_{1}, y_{2}, \ldots, y_{d}\right)$ then we note that $A$ has 1 's in positions $\left(y_{1}, y_{2}, \ldots, y_{d}(j)\right)$ for $j \in[q]$ where $y_{d}=y_{d}(1)$ and $y_{d}(1)<y_{d}(2)<\cdots<y_{d}(q)$. It is now straightforward to show that choosing indices $I(1)=\left\{y_{1}-1, y_{1}\right\}, I(2)=\left\{y_{2}-1, y_{2}\right\}$, $\ldots, I(d-1)=\left\{y_{d-1}-1, y_{d}\right\}$ and $I(d)=\left\{y_{d}(1), y_{d}(2), \ldots, y_{d}(q-1)\right\}$ yields a $\overbrace{3,3, \ldots, 3}^{d-1}, q$ split. We can show that $\sigma_{1}(B) \leq \sigma_{1}(A)-\sigma_{1}\left(A^{\prime}\right)$, hence if $\sigma_{1}(A)>\sigma_{1}(B)$, then $A$ would have the desired split.

\section{Submatrices of $T \times T$}

We show how to exploit the results about splits in the context of forbidden configurations but begin with the following elementary argument.

Proof of Proposition 1: We note that any row from $E_{i}$ contains [0 1$]$ and we define $K_{2}=\left[\begin{array}{ll}0 & 1\end{array}\right] \times\left[\begin{array}{ll}0 & 1\end{array}\right]$. None of our 2-rowed product terms $I, T, I^{c}$ contain $K_{2}$. Two rows of $F$ chosen from two different terms of the $(u+v+w)$-fold product, will necessarily contain $K_{2}$. This implies that if $F$ is contained in the $a$-fold product $\overbrace{I_{m / a} \times \cdots \times I_{m / a}}^{p}$ $\times \overbrace{T_{m / a} \times \cdots \times T_{m / a}}^{q} \times \overbrace{I_{m / a}^{c} \times \cdots \times I_{m / a}^{c}}^{r}$, then each product term $I_{m / a}, T_{m / a}, I_{m / a}^{c}$ has at most 2 rows of $F$ and if it has two rows then they come from the same 2-rowed product term $E_{i}$ of $F$. Of the three matrices $I_{m / a}, T, I^{c}$, we note that we can find $E_{1}$ only in $I_{m / a}$, $E_{2}$ only in $T_{m / a}$ and $E_{3}$ only in $I_{m / a}^{c}$.

Proof of Theorem 3 that $f\left(E_{2} \times E_{2}, T_{m / 2} \times T_{m / 2}\right) \leq 2 m$. Let $F=E_{2} \times E_{2}$. Recall the $i$ th column of $T_{k}$ is the column with 1 's in rows $1,2, \ldots, i$ and 0 's in the remaining rows. Let $A$ be an $m$-rowed submatrix of $T_{m / 2} \times T_{m / 2}$. We can create an $m / 2 \times m / 2(0,1)$-matrix $B$ from $A$ by placing a 1 in position $(r, c)$ if $A$ contains the column obtained from the $r$ th column of $T_{m / 2}$ placed on top of the $c$ th column of $T_{m / 2}$ namely the column with 1's only in rows $1,2, \ldots r$ and $m+1, m+2, \ldots, m+c$. We note that $\|A\|$ is $\sigma_{1}(B)$.

We claim that $A$ has $F$ as a configuration if and only if $B$ has a 3,3 split. The only way for a submatrix of $T_{m / 2} \times T_{m / 2}$ to be a row and column permutation of $F$ is to lie in rows $r_{1}, r_{2}, m / 2+c_{1}, m / 2+c_{2}$ for some choices $2 \leq r_{1}<r_{2} \leq m / 2$ and $2 \leq c_{1}<c_{2} \leq m / 2$ (using the argument of Proposition 1 for $E_{2} \times E_{2}$ and noting that first row of $T_{m / 2}$ is 1 's). We have that any two rows of the upper triangular matrix $T_{m / 2}$ (not including the first) have a copy of $E_{2}$. We note that the $t$ th column of $T_{m / 2}$ on rows $r_{1}, r_{2}$ (with $r_{1}<r_{2}$ ) has

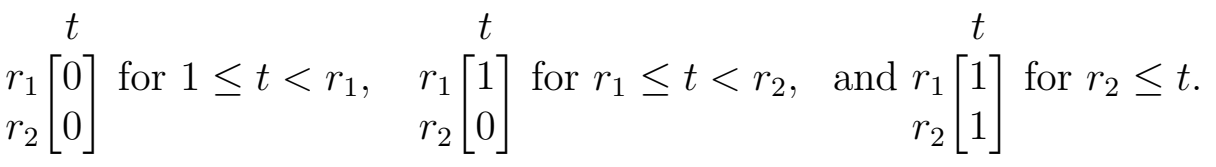


Assume $A$ has a copy of $F$ in the 4 rows $r_{1}, r_{2}, m / 2+c_{1}, m / 2+c_{2}$. We discover that the nine columns of $F$ would correspond to nine 1's, one 1 in each of the nine blocks in the 3,3 split of $B$ given by $I(1)=\left\{r_{1}-1, r_{2}-1\right\}$ and $I(2)=\left\{c_{1}-1, c_{2}-1\right\}$ (notation from Section 2). Similarly a 3,3 split of $B$ yields a copy of $F$ in $A$. We now appeal to the bound in Theorem 7 .

An immediate generalization is the following.

Lemma 1. $f(\overbrace{E_{2} \times E_{2} \times \cdots \times E_{2}}^{d}, \overbrace{T_{m / d} \times T_{m / d} \times \cdots \times T_{m / d}}^{d})$ is $\Theta\left(m^{d-1}\right)$.

Proof: Let $A$ be an $m$-rowed submatrix of $\overbrace{T_{m / d} \times T_{m / d} \times \cdots \times T_{m / d}}^{d}$. We generalize the proof of Theorem 7 and encode $A$ as an $d$-dimension (0,1)-array $B$ where we place a 1 in $B$ in position $\left(a_{1}, a_{2}, \ldots, a_{d}\right)$ if $A$ has a column consisting of the $a_{1}$ st column of $T_{m / d}$ on top of the $a_{2}$ nd column of $T_{m / d}$ etc on top of the $a_{d}$ th column of $T_{m / d}$. As before, $\|A\|=\sigma_{1}(B)$. We verify that $A$ will have the configuration of the $d$-fold product $E_{2} \times E_{2} \times \cdots \times E_{2}$ if and only if $B$ has a $\overbrace{3,3, \ldots, 3}^{d}$ split. We have an exact bound from Theorem 9 if needed.

A further generalization considers the matrix $E_{2}(k)=\left[\mathbf{0}_{k} \mid T_{k}\right]$ (the columns of $k$-rowed submatrices of $\left.T_{m}\right)$.

Lemma 2. We have that

$$
f(\overbrace{E_{2}(k) \times E_{2}(k) \times \cdots \times E_{2}(k)}^{d}, \overbrace{T_{m / d} \times T_{m / d} \times \cdots \times T_{m / d}}^{d})
$$

is equal to

$$
g(m / d, m / d, \ldots, m / d ; k+1, k+1, \ldots, k+1)
$$

and so is $\Theta\left(m^{d-1}\right)$.

Proof: We use the $d$-dimensional generalization of splits Klazar,Marcus [14] and Balogh, Bollabás, Morris [8] where the $d$-fold product $E_{2}(k) \times E_{2}(k) \times \cdots \times E_{2}(k)$ will correspond to a $\overbrace{k+1, k+1, \ldots, k+1}^{d}$ split.

A rather interesting version of Theorem 3 and Lemma 1 that uses the idea of 'peeling' from Theorem 7 is the following.

Lemma 3. Let $p \geq 3$. Then $f\left(E_{2} \times E_{2}, T_{m / p} \times T_{m / p} \times \cdots \times T_{m / p}\right) \leq \frac{m}{p} \cdot 4^{p-1}$.

Proof: Let $F=E_{2} \times E_{2}$. We will show that $f\left(E_{2} \times E_{2}, T_{\hat{m}} \times T_{\hat{m}} \times \cdots \times T_{\hat{m}}\right) \leq 4^{p-1} \hat{m}$. We consider $A$ as an $\hat{m} \times \hat{m} \cdots \times \hat{m})$ p-dimensional $(0,1)$-array $B$ as follows. Let $x_{1}, x_{2}, \ldots, x_{p}$ be the $p$ coordinate directions in $B$. The entries in coordinate direction $x_{i}$ are indexed by the columns of $T_{m / p}$ in the given order. We note that $\|A\|=\sigma_{1}(B)$.

We first handle the case $p=3$. By Theorem 3 , we have that for $i=1,2,3, \sigma_{1}\left(\operatorname{proj}_{i}(B)\right)$ is at most $4 \hat{m}$. In fact if $\sigma_{1}\left(\operatorname{proj}_{i}(B)\right)>4 \hat{m}$ then we have a 3,3 split in $\operatorname{proj}_{i}(B)$ and that yields $F$ in $A$ where no rows of $F$ come from the $i$ th term $T_{\hat{m}}$ of the product and 2 rows of $F$ come from another $T_{\hat{m}}$ of the product and the other 2 rows of $F$ come from remaining part $T_{\hat{m}}$. 
Now proceed to form a matrix $B^{\prime}$ from $B$ by deleting from $B$ in turn the top 1 in each line in the direction $x_{3}$ and then deleting the bottom 1 in each line in the direction $x_{2}$ and finally deleting the top two entries in each line in the direction $x_{1}$. We have

$$
\sigma_{1}(B) \leq \sigma_{1}\left(B^{\prime}\right)+\sigma_{1}\left(\operatorname{proj}_{3}(B)\right)+\sigma_{1}\left(\operatorname{proj}_{2}(B)\right)+2 \sigma_{1}\left(\operatorname{proj}_{1}(B)\right) \leq \sigma_{1}\left(B^{\prime}\right)+4 \cdot 4 \hat{m},
$$

where we are using the size of the projections to upper bound the number of deleted 1's. Let $\mathbf{y}_{1}$ be a 1 of $B^{\prime}$. Then, by our construction, there are 2 further 1 's of $B$ in positions $\mathbf{y}_{2}, \mathbf{y}_{3}$ with $x_{1}\left(\mathbf{y}_{1}\right)<x_{1}\left(\mathbf{y}_{2}\right)<x_{1}\left(\mathbf{y}_{3}\right), x_{2}\left(\mathbf{y}_{1}\right)=x_{2}\left(\mathbf{y}_{2}\right)=x_{2}\left(\mathbf{y}_{3}\right)$ and $x_{3}\left(\mathbf{y}_{1}\right)=x_{3}\left(\mathbf{y}_{2}\right)=$ $x_{3}\left(\mathbf{y}_{3}\right)$. For each $\mathbf{y}_{j}$ for $j=1,2,3$, we will have two 1 's in positions $\mathbf{y}_{j}^{\prime}, \mathbf{y}_{j}^{\prime \prime}$ of $B$ where $\mathbf{y}_{j}^{\prime}$ agrees with $\mathbf{y}_{j}$ except in coordinate $x_{2}$ where $x_{2}\left(\mathbf{y}_{j}^{\prime}\right)<x_{2}\left(\mathbf{y}_{j}\right)$ and $\mathbf{y}_{j}^{\prime \prime}$ agrees with $\mathbf{y}_{j}$ except in coordinate $x_{3}$ where $x_{3}\left(\mathbf{y}_{j}\right)<x_{3}\left(\mathbf{y}_{j}^{\prime \prime}\right)$. Then these 91 's in $B$ correspond to a copy of $F$ in $A$ as follows. Note that column $t$ of $T_{\hat{m}}$ has a 0 in row $r$ if and only if $t<r$. We choose two values $a=x_{1}\left(\mathbf{y}_{2}\right)$ and $b=a+1$ for coordinate $x_{1}$ so that when we consider the columns of $A$ corresponding to $\mathbf{y}_{1}$ (and $\mathbf{y}_{1}^{\prime}, \mathbf{y}_{1}^{\prime \prime}$ respectively ), $\mathbf{y}_{2}$ (and $\mathbf{y}_{2}^{\prime}, \mathbf{y}_{2}^{\prime \prime}$ resp.), $\mathbf{y}_{3}$ (and $\mathbf{y}_{3}^{\prime}, \mathbf{y}_{3}^{\prime \prime}$ resp.) we have

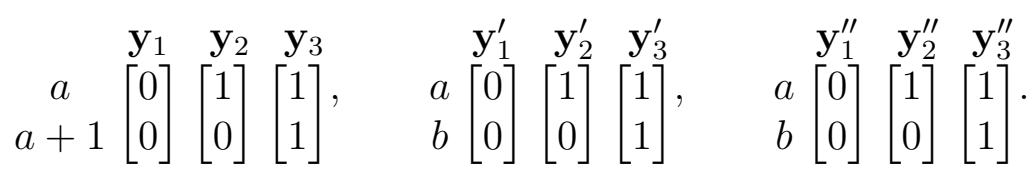

Note that for each $j=1,2,3$, we have $x_{2}\left(\mathbf{y}_{j}^{\prime}\right)<x_{2}\left(\mathbf{y}_{j}\right)=x_{2}\left(\mathbf{y}_{j}^{\prime \prime}\right)$ and $x_{3}\left(\mathbf{y}_{j}^{\prime}\right)=x_{3}\left(\mathbf{y}_{j}\right)<$ $x_{3}\left(\mathbf{y}_{j}^{\prime \prime}\right)$. We can choose a value $c=x_{2}\left(\mathbf{y}_{1}\right)$ for $x_{2}$ and a value $d=x_{3}\left(\mathbf{y}_{1}\right)+1$ for $x_{3}$ (independent of $j$ ) so that in $A$, the columns corresponding to $\mathbf{y}_{j}, \mathbf{y}_{j}^{\prime}, \mathbf{y}_{j}^{\prime \prime}$ have

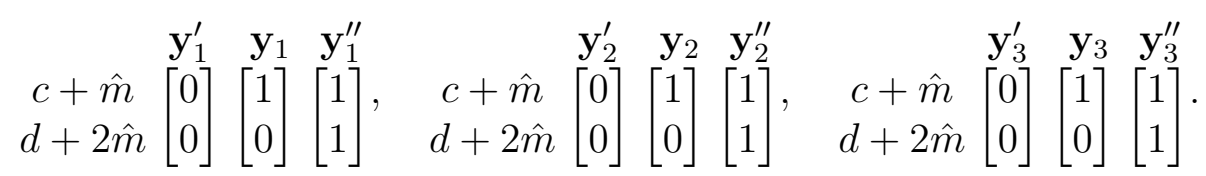

This yields a copy of $F$ in $A$ in rows $a, b, c+\hat{m}, d+2 \hat{m}$, a contradiction. We deduce that $\sigma_{1}\left(B^{\prime}\right)=0$ and hence $\sigma_{1}(B) \leq 4^{2} \hat{m}$, concluding the proof for $p=3$.

For $p \geq 4$, we proceed in a somewhat similar fashion but focussing on the first 4 coordinates. By induction on $p, \sigma_{1}\left(\operatorname{proj}_{i}(B)\right)$ is at most $4^{p-2} \hat{m}$. We form a matrix $B^{\prime}$ from $B$ by deleting from $B$, if possible, the top 1 in each line in the direction $x_{4}$ and then deleting the bottom 1 in each line in the direction $x_{3}$ and then deleting the top 1 in each line in the direction $x_{2}$ finally deleting the bottom 1 in each line in the direction $x_{1}$. We have

$$
\sigma_{1}(B) \leq \sigma_{1}\left(B^{\prime}\right)+\sum_{i=1}^{4} \sigma_{1}\left(\operatorname{proj}_{i}(B)\right) \leq \sigma_{1}\left(B^{\prime}\right)+4 \cdot 4^{p-2} \hat{m}=4^{p-1} \hat{m}
$$

using the fact that $\operatorname{proj}_{i}(B)$ is a $(\mathrm{p}-1)$-dimensional array and induction on $p$. Let $\mathbf{y}_{1}$ be an 1 of $B^{\prime}$. Then, by our construction, there are 2 further 1's of $B$ in positions $\mathbf{y}_{2}, \mathbf{y}_{3}$ with $x_{1}\left(\mathbf{y}_{2}\right)<x_{1}\left(\mathbf{y}_{1}\right)$ and $x_{i}\left(\mathbf{y}_{2}\right)=x_{i}\left(\mathbf{y}_{1}\right)$ for $i \neq 1$, and $x_{2}\left(\mathbf{y}_{1}\right)<x_{2}\left(\mathbf{y}_{3}\right)$ and $x_{i}\left(\mathbf{y}_{1}\right)=x_{i}\left(\mathbf{y}_{3}\right)$ for $i \neq 2$. For each $\mathbf{y}_{j}$ we will have two 1's in positions $\mathbf{y}_{j}^{\prime}, \mathbf{y}_{j}^{\prime \prime}$ of $B$ where $\mathbf{y}_{j}^{\prime}$ agrees with $\mathbf{y}_{j}$ except in coordinate $x_{3}$ where $x_{3}\left(\mathbf{y}_{j}^{\prime}\right)<x_{3}\left(\mathbf{y}_{j}\right)$ and $\mathbf{y}_{j}^{\prime \prime}$ agrees with $\mathbf{y}_{j}$ except in coordinate $x_{4}$ where $x_{4}\left(\mathbf{y}_{j}\right)<x_{4}\left(\mathbf{y}_{j}^{\prime \prime}\right)$. Then these 91 's in $B$ correspond to a copy of $F$. In particular we can choose values $a=x_{1}\left(\mathbf{y}_{1}\right), b=x_{2}\left(\mathbf{y}_{1}\right)+1$ so that in $A$ the columns contain

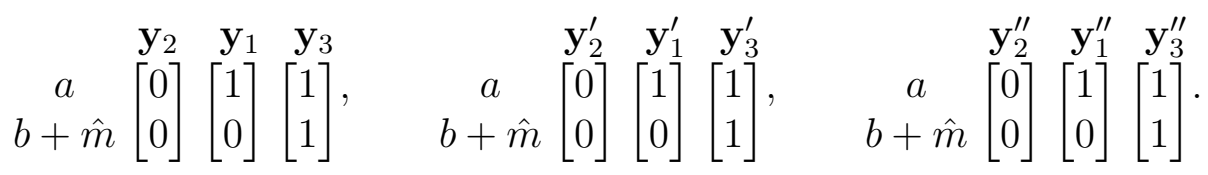


As above we can choose values $c=x_{3}\left(\mathbf{y}_{1}\right)$ and $d=x_{4}\left(\mathbf{y}_{1}\right)+1$ and obtain a copy of $F$ in rows $a, b+\hat{m}, c+2 \hat{m}, d+3 \hat{m}$ of $A$ from the 9 columns of $A$ given by the 91 's of $B$, a contradiction.

We deduce that $\sigma_{1}\left(B^{\prime}\right)=0$ and hence $\sigma_{1}(B) \leq 4^{p-1} \hat{m}$

Some growth in the bound with respect to $p$ is to be expected since forb $\left(m, E_{2} \times E_{2}\right)$ is $\Theta\left(m^{3}\right)$.

\section{Submatrices of $I \times I$}

Proof of Theorem 1 that $f\left(E_{1} \times E_{1}, I_{m / 2} \times I_{m / 2}\right)$ is $\Theta\left(m^{3 / 2}\right)$. Let $F=E_{1} \times E_{1}$. Let $A$ be a submatrix of $I_{m / 2} \times I_{m / 2}$ with no configuration $F$. We consider $A$ as an $(m / 2) \times(m / 2)$ $(0,1)$-matrix $B$ whose rows are indexed by the columns of $I_{m / 2}$ and whose columns are indexed by the columns of $I_{m / 2}$. Then $\|A\|=\sigma_{1}(B)$. Note that the $r$ th row of $I_{m / 2}$ has a 1 in column $r$ of $I_{m / 2}$ and 0's in all other columns. Thus a $2 \times 2$ submatrix of 41 's in $B$ in rows $r_{1}, r_{2}$ and columns $c_{1}, c_{2}$ will yield $I_{2} \times I_{2}$ in the associated 4 columns of $A$ (we let $(r, c)$ label the column of $A$ associated with the 1 in position $(r, c)$ of $B)$ :

$\left(r_{1}, c_{1}\right)$
$r_{1}$
$r_{2}$
$c_{1}$
$c_{2}$$\left[\begin{array}{cccc}1 & \left.r_{1}, c_{2}\right) & \left(r_{2}, c_{1}\right) & \left(r_{2}, c_{2}\right) \\ 0 & 0 & 0 & 0 \\ 1 & 0 & 1 & 1 \\ 0 & 1 & 0 & 1\end{array}\right]$

The remaining 5 columns of $E_{1} \times E_{1}$ are less structured. Four rows of $A$ contains $F$ if and only if 2 rows of $A$ (say $r_{1}, r_{2}$ chosen from the first $m / 2$ rows of $A$ ) contain the first two rows of $F$ corresponding to a copy of $E_{1}$ in $E_{1} \times E_{1}$ and 2 rows of $A$ (say $c_{1}+m / 2, c_{2}+m / 2$ chosen from the last $m / 2$ ) contain the third and fourth rows of $F$ (and the other $E_{1}$ in $E_{1} \times E_{1}$ ). Now $A$ having 9 columns containing $F$ correspond to $B$ having 9 1's as follows: a $2 \times 2$ submatrix of 1 's in rows $r_{1}, r_{2}$ and columns $c_{1}, c_{2}$ and one more 1 in each row of the $2 \times 2$ submatrix and one more 1 in each column of the submatrix and one more 1 in neither of the two chosen rows or two chosen columns. To see this consider a 1 in position $r_{1}, c$ of $B$ with $c \neq c_{1}, c_{2}$. Then column $\left(r_{1}, c\right)$ of $A$ will have $\left[\begin{array}{l}1 \\ 0\end{array}\right]$ in rows $r_{1}, r_{2}$ and $\left[\begin{array}{l}0 \\ 0\end{array}\right]$ in rows $c_{1}+m / 2, c_{2}+m / 2$. A 1 in position $r, c$ of $B$ with $r \neq r_{1}, r_{2}$ and $c \neq c_{1}, c_{2}$ will yield a column $(r, c)$ in $A$ that is all 0's on row $r_{1}, r_{2}, c_{1}+m / 2, c_{2}+m / 2$.

Assume $\|A\|=\sigma_{1}(B) \geq\left(\frac{m}{2}\right)^{3 / 2}+2.5 m$. We initially process $B$ by deleting any row or column with at most two 1's (and hence up to $2 \cdot 2 \cdot \frac{m}{2} 1$ 's) repeating the deletion process if necessary so that the resulting matrix $\bar{B}$ has row and column sums at least 3. We note that $\sigma_{1}(B) \leq \sigma_{1}(\bar{B})+2 m$. Then $\sigma_{1}(\bar{B}) \geq\left(\frac{m}{2}\right)^{3 / 2}+m / 2$. We now appeal to Kövari, Sós and Turán [15] for a solution of Zarankiewicz' problem and deduce that $\bar{B}$ has a $2 \times 2$ block of 1 's and then $\bar{B}$ has the configuration of 91 's yielding $F$ in $A$. Thus $\|A\|<\left(\frac{m}{2}\right)^{3 / 2}+2.5 m$.

A construction using projective planes [15] establishes $f\left(E_{1} \times E_{1}, I_{m / 2} \times I_{m / 2}\right)$ is $\Omega\left(m^{3 / 2}\right)$.

Problem 1. Determine $f\left(E_{1} \times E_{1} \times E_{1}, I_{m / 3} \times I_{m / 3} \times I_{m / 3}\right)$.

The crux of this problem would be determining the maximum number of 1's in a 3dimensional $(m / 3) \times(m / 3) \times(m / 3)(0,1)$-array which has no $2 \times 2 \times 2$ subarray of 81 's. 
Erdős [9] has obtained a bound $O\left(m^{11 / 4}\right)$ for this but only a $\Omega\left(m^{5 / 2}\right.$ construction. Note the sharp contrast with results such as Theorem 3, Lemma 1, Lemma 3.

\section{Submatrices of $I \times T$}

It is useful to state a result about arrangements of 1's in a 2-dimensional array.

Lemma 4. Let $B$ be an $\hat{m} \times \hat{m}$ matrix with $\sigma_{1}(B)>4 \hat{m}$. Then there are 91 's in $B$ in the following configuration consisting of three lines of three 1 's:

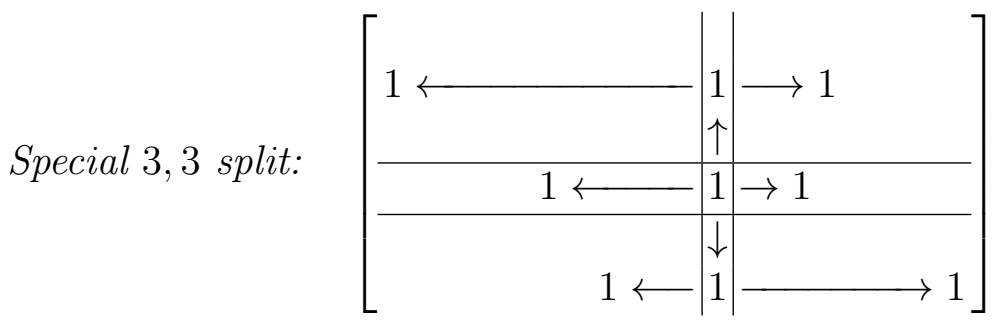

If the central 1 is in position $\left(r_{1}, c_{1}\right)$ then we have a 3,3 split using $I(1)=\left\{r_{1}-1, r_{1}\right\}$ and $I(2)=\left\{c_{1}-1, c_{1}\right\}$ (notation from Section 2)

Proof: We may argue that $\sigma_{1}(B) \leq 4 \hat{m}$ as follows. Form a matrix $B^{\prime}$ from $B$ by deleting from $B$, if possible, the top and bottom 1 in each row (a line in the direction $x_{2}$ ) and then deleting, if possible, the top and bottom 1 in each column ( a line in the direction $\left.x_{1}\right)$. We have that $\sigma_{1}(B) \leq \sigma_{1}\left(B^{\prime}\right)+4 \hat{m}$.

If $\sigma_{1}\left(B^{\prime}\right)>0$, then select a 1 in $B^{\prime}$ in position $\mathbf{y}_{1}$. Then there are two 1 's in $B$ in positions $\mathbf{y}_{2}, \mathbf{y}_{3}$ in the same column as $\mathbf{y}_{1}, \mathbf{y}_{2}$ lying below $\mathbf{y}_{1}$ and $\mathbf{y}_{3}$ lying above. Then for each $\mathbf{y}_{i}$ there are two additional 1's in $B$ positions $\mathbf{y}_{i}^{\prime}, \mathbf{y}_{i}^{\prime \prime}$ lying to the left and to the right of $\mathbf{y}_{i}$ in the same row as $\mathbf{y}_{i}$.

Now $\mathbf{y}_{1}=\left(r_{1}, c_{1}\right)$ is the central 1 and this yields a 3,3 split as described.

Proof of Theorem 2 that $f\left(E_{1} \times E_{2}, I_{m / 2} \times T_{m / 2}\right) \leq 2 m$. Let $F=E_{1} \times E_{2}$. Let $A$ be an $m$-rowed submatrix of $I_{m / 2} \times T_{m / 2}$ with no $F$. We consider $A$ as an $(m / 2) \times(m / 2)$ $(0,1)$-matrix $B$ whose rows are indexed by the columns of $I_{m / 2}$ and whose columns are indexed by the columns of $T_{m / 2}$ in the usual order. We note that $\|A\|=\sigma_{1}(B)$.

By Lemma 4, if $\sigma_{1}(B)>2 m$, we can find an arrangement of 91 's as in (3). Let $\mathbf{y}_{1}$ denote the central 1 with $\mathbf{y}_{2}$ denoting the 1 below $\mathbf{y}_{1}$ and $\mathbf{y}_{3}$ denoting the 1 above. Let the position of the three 1's be $\mathbf{y}_{i}=\left(r_{i}, c_{1}\right)$ for $i=1,2,3$. Now $r_{i}=x_{1}\left(\mathbf{y}_{i}\right)=x_{1}\left(\mathbf{y}_{i}^{\prime}\right)=x_{1}\left(\mathbf{y}_{i}^{\prime \prime}\right)$ for $i=1,2,3$ and so in the columns of $A$ corresponding to the 91 's we find

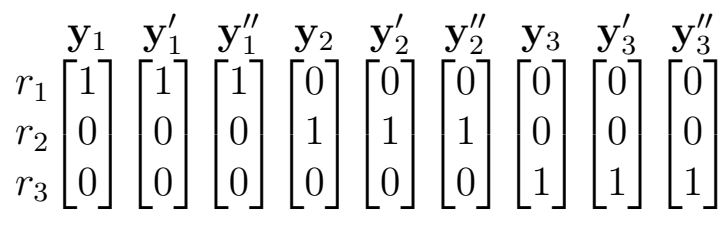

We now use the 3,3 split idea. Let $c_{1}=x_{2}\left(\mathbf{y}_{1}\right)=x_{2}\left(\mathbf{y}_{2}\right)=x_{2}\left(\mathbf{y}_{3}\right)$. Column $c_{1}$ of $T_{m / 2}$ has $\begin{gathered}c_{1} \\ c_{1}+1\end{gathered}\left[\begin{array}{l}1 \\ 0\end{array}\right]$. Also any column $a$ of $T_{m / 2}$ with $a<c_{1}$ has ${ }_{c_{1}+1}^{c_{1}}\left[\begin{array}{l}0 \\ 0\end{array}\right]$ and any column $b$ of $T_{m / 2}$ with $c_{1}<b$ has $\begin{gathered}c_{1} \\ c_{1}+1\end{gathered}\left[\begin{array}{l}1 \\ 1\end{array}\right]$. Note that $x_{2}\left(\mathbf{y}_{j}^{\prime}\right)<c_{1}=x_{2}\left(\mathbf{y}_{j}\right)<x_{2}\left(\mathbf{y}_{j}^{\prime \prime}\right)$ for $j=1,2,3$. Then we find in $A$ in the 9 columns: 


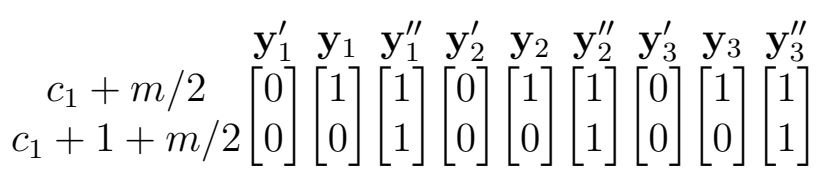

Thus in rows $r_{1}, r_{2}, c_{1}+m / 1, c_{1}+1+m / 2$ of $A$ we find $E_{1} \times E_{2}$. This contradiction establishes the bound.

The construction $A=I_{m / 2} \times \mathbf{1}_{m / 2}$ avoids $F$ and has $\Omega(m)$ columns.

It is useful to give one result about arrangements of 1's in a 3-dimensional array.

Lemma 5. Let $C$ be an $\hat{m} \times \hat{m} \times \hat{m}$ 3-dimensional $(0,1)$-array with more than $6 \hat{m}^{2} 1$ 's. Then there are 27 1's as follows. There are three values $a, b, c$ for $x_{1}$ coordinate such that the three planes $x_{1}=a, x_{1}=b$ and $x_{1}=c$ of $C$ each contains 9 points. The 9 points in each plane form a special 3,3 split as in (3) with the central 1 in each of the three planes having the same $x_{2}, x_{3}$ coordinates.

Proof: Form a matrix $C^{\prime}$ from $C$ by deleting from $C$, if possible, the top and bottom 1 in each line in direction $x_{3}$ then the top and bottom 1 in each line in the direction $x_{2}$ and then the top two 1's in each line in the direction $x_{1}$. We obtain

$$
\sigma_{1}(C) \leq \sigma_{1}\left(C^{\prime}\right)+6 \hat{m}^{2}
$$

If $C^{\prime}$ has a 1 in position $\mathbf{y}_{1}$, we can find 27 1's yielding a special 3,3,3 split as follows. There are 21 's of $C$ in positions $\mathbf{y}_{2}, \mathbf{y}_{3}$ with $x_{1}\left(\mathbf{y}_{1}\right)<x_{1}\left(\mathbf{y}_{2}\right)<x_{1}\left(\mathbf{y}_{3}\right), x_{2}\left(\mathbf{y}_{1}\right)=x_{2}\left(\mathbf{y}_{2}\right)=$ $x_{2}\left(\mathbf{y}_{3}\right)$ and $x_{3}\left(\mathbf{y}_{1}\right)=x_{3}\left(\mathbf{y}_{2}\right)=x_{3}\left(\mathbf{y}_{3}\right)$. Then there are 1's of $C$ in positions $\mathbf{x}_{j}, \mathbf{z}_{j}$ for $j=1,2,3$, where $x_{2}\left(\mathbf{x}_{j}\right)<x_{2}\left(\mathbf{y}_{j}\right)<x_{2}\left(\mathbf{z}_{j}\right)$ and $x_{1}\left(\mathbf{x}_{j}\right)=x_{1}\left(\mathbf{y}_{j}\right)=x_{1}\left(\mathbf{z}_{j}\right), x_{3}\left(\mathbf{x}_{j}\right)=$ $x_{3}\left(\mathbf{y}_{j}\right)=x_{3}\left(\mathbf{z}_{j}\right)$. Now for each choice $\mathbf{v} \in\{\mathbf{x}, \mathbf{y}, \mathbf{z}\}$, we obtain positions $\mathbf{v}_{j}^{\prime}, \mathbf{v}_{j}^{\prime \prime}$ for $j=1,2,3$ with $x_{3}\left(\mathbf{v}_{j}^{\prime}\right)<x_{3}\left(\mathbf{v}_{j}\right)<x_{3}\left(\mathbf{v}_{j}^{\prime \prime}\right)$ and $x_{1}\left(\mathbf{v}_{j}^{\prime}\right)=x_{1}\left(\mathbf{v}_{j}\right)=x_{1}\left(\mathbf{v}_{j}^{\prime \prime}\right), x_{2}\left(\mathbf{v}_{j}^{\prime}\right)=x_{2}\left(\mathbf{v}_{j}\right)=x_{2}\left(\mathbf{v}_{j}^{\prime \prime}\right)$. In particular there are three planes $x_{1}=a, x_{1}=b, x_{1}=c$ each with 91 's and each plane has a special 3,3 split as in (3) with the central 1's of each plane (namely $\mathbf{y}_{1}, \mathbf{y}_{2}, \mathbf{y}_{3}$ ) having the same $x_{2}, x_{3}$ coordinates. The horizontal direction in (3) corresponds to the $x_{3}$ direction.

Lemma 6. $f\left(E_{1} \times E_{2} \times E_{2}, I_{m / 3} \times T_{m / 3} \times T_{m / 3}\right)$ is $\Theta\left(m^{2}\right)$.

Proof: Let $A$ be an $m$-rowed submatrix of $I_{m / 3} \times T_{m / 3} \times T_{m / 3}$ with no configuration $E_{1} \times E_{2} \times E_{2}$. As above, we translate $A$ into a 3 -dimensional array $B$ with $\|A\|=\sigma_{1}(B)$.

Now by Lemma 5 , if $\sigma_{1}(B)>6(\mathrm{~m} / 3)^{2}$ there will be 27 's in $B$ as described and we use the notation of the proof. This will yield a copy of $E_{1} \times E_{2} \times E_{2}$ in $A$. Let the central 1 in each plane be in position $\mathbf{y}_{i}$ for $i=1,2,3$ with $x_{1}\left(\mathbf{y}_{1}\right)=r_{1}<x_{1}\left(\mathbf{y}_{2}\right)=r_{2}<x_{1}\left(\mathbf{y}_{3}\right)=r_{3}$. Then there are 1's of $B$ in positions $\mathbf{x}_{j}, \mathbf{z}_{j}$ for $j=1,2,3$, where $x_{2}\left(\mathbf{x}_{j}\right)<x_{2}\left(\mathbf{y}_{j}\right)<$ $x_{2}\left(\mathbf{z}_{j}\right)$ and $x_{1}\left(\mathbf{x}_{j}\right)=x_{1}\left(\mathbf{y}_{j}\right)=x_{1}\left(\mathbf{z}_{j}\right), x_{3}\left(\mathbf{x}_{j}\right)=x_{3}\left(\mathbf{y}_{j}\right)=x_{3}\left(\mathbf{z}_{j}\right)$. Now for each choice $\mathbf{v} \in\{\mathbf{x}, \mathbf{y}, \mathbf{z}\}$, we obtain positions $\mathbf{v}_{j}^{\prime}, \mathbf{v}_{j}^{\prime \prime}$ for $j=1,2,3$ with $x_{3}\left(\mathbf{v}_{j}^{\prime}\right)<x_{3}\left(\mathbf{v}_{j}\right)<x_{3}\left(\mathbf{v}_{j}^{\prime \prime}\right)$ and $x_{1}\left(\mathbf{v}_{j}^{\prime}\right)=x_{1}\left(\mathbf{v}_{j}\right)=x_{1}\left(\mathbf{v}_{j}^{\prime \prime}\right), x_{2}\left(\mathbf{v}_{j}^{\prime}\right)=x_{2}\left(\mathbf{v}_{j}\right)=x_{2}\left(\mathbf{v}_{j}^{\prime \prime}\right)$. Now $r_{i}=x_{1}\left(\mathbf{v}_{i}^{\prime}\right)=x_{1}\left(\mathbf{v}_{i}\right)=x_{1}\left(\mathbf{v}_{i}^{\prime \prime}\right)$ for $i=1,2,3$ and also all choices $\mathbf{v} \in\{\mathbf{x}, \mathbf{y}, \mathbf{z}\}$. In the columns of $A$ corresponding to the 27 1's we find

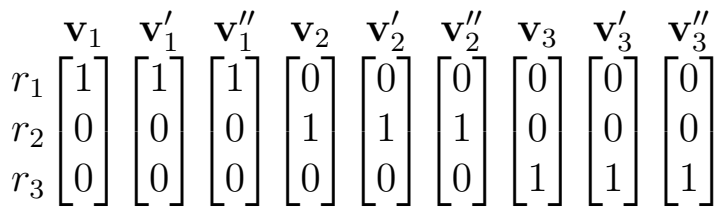


Note that in rows $r_{1}, r_{2}$, we see copies of $E_{1}$. Now let $c_{2}=x_{2}\left(\mathbf{y}_{1}\right), c_{3}=x_{3}\left(\mathbf{y}_{1}\right)$. Recall that $x_{2}\left(\mathbf{y}_{1}\right)=x_{2}\left(\mathbf{y}_{2}\right)=x_{2}\left(\mathbf{y}_{3}\right)=x_{2}\left(\mathbf{y}_{j}^{\prime}\right)=x_{2}\left(\mathbf{y}_{j}^{\prime \prime}\right)$ for all $j=1,2,3$. These 91 's lie on the plane with $x_{2}$ coordinate $c_{2}$. The same need not be true if we replace $\mathbf{y}$ by $\mathbf{x}$ or $\mathbf{z}$ but, for each choice of $j \in\{1,2,3\}$, we have $x_{2}\left(\mathbf{x}_{j}\right)=x_{2}\left(\mathbf{x}_{j}^{\prime}\right)=x_{2}\left(\mathbf{x}_{j}^{\prime \prime}\right)<c_{2}$ and $c_{2}<x_{2}\left(\mathbf{z}_{j}\right)=x_{2}\left(\mathbf{z}_{j}^{\prime}\right)=x_{2}\left(\mathbf{z}_{j}^{\prime \prime}\right)$. Column $c_{2}$ of $T_{m / 2}$ has $\begin{gathered}c_{2} \\ c_{2}+1\end{gathered}\left[\begin{array}{l}1 \\ 0\end{array}\right]$. Also any column $a$ of $T_{m / 2}$ with $a<c_{2}$ has $\begin{gathered}c_{2} \\ c_{2}+1\end{gathered}\left[\begin{array}{l}0 \\ 0\end{array}\right]$ and any column $b$ of $T_{m / 2}$ with $c_{2}<b$ has $\begin{gathered}c_{2} \\ c_{2}+1\end{gathered}\left[\begin{array}{l}1 \\ 1\end{array}\right]$. Recalling that $x_{2}\left(\mathbf{x}_{j}\right)<c_{2}=x_{2}\left(\mathbf{y}_{j}\right)<x_{2}\left(\mathbf{z}_{j}\right)$, we have for each $j=1,2,3$ in $A$ in rows $c_{2}+m / 1, c_{2}+1+m / 3$ the following in the columns of corresponding to the 271 's while letting $j=1,2,3$ :

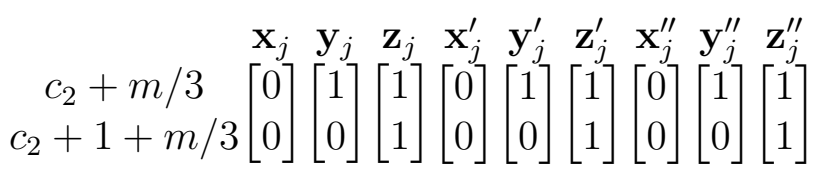

We use the same ideas noting that $x_{3}\left(\mathbf{v}^{\prime}\right)<x_{3}(\mathbf{v})<x_{3}\left(\mathbf{v}^{\prime \prime}\right)$ for each $j=1,2,3$ and each choice $\mathbf{v} \in\{\mathbf{x}, \mathbf{y}, \mathbf{z}\}$. In this case there are 91 's with the same $x_{3}$ coordinate as $\mathbf{y}_{1}$ (the positions $\mathbf{v}_{j}$ for all choices $j=1,2,3$ and $\mathbf{v} \in\{\mathbf{x}, \mathbf{y}, \mathbf{z}\}$ ). With $c_{3}=x_{3}\left(\mathbf{y}_{1}\right)$ we have the following in the columns corresponding to the 271 's while letting $j=1,2,3$ :

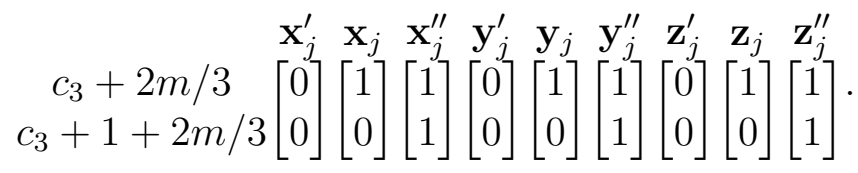

We now have a copy of $E_{1} \times E_{2} \times E_{2}$ in $A$, a contradiction and so $\|A\| \leq 6(\mathrm{~m} / 3)^{2}$.

Let $\alpha$ being first column of $I_{m / 3}$. Using Proposition 1, the construction $\alpha \times T_{m / 3} \times T_{m / 3}$ avoids $F$ and has $\Theta\left(m^{2}\right)$ columns.

Using an analogous argument one obtains

Lemma 7. $f(E_{1} \times \overbrace{E_{2} \times \cdots \times E_{2}}^{p-1}, I_{m / p} \times \overbrace{T_{m / p} \times \cdots \times T_{m / p}}^{p-1})$ is $\Theta\left(m^{p-1}\right)$.

\section{Proof of the unexpected bound}

Proof of Theorem 4. Let $A$ be an $m$-rowed simple matrix with no configurations $\left\{I_{2} \times I_{2}, T_{2} \times T_{2}\right\}$. Our standard decomposition on row $r$ considers deleting row $r$ from $A$ and reordering the columns as below with $C_{r}$ consisting of the columns which are repeated in the matrix obtained from $A$ by deleting row $r$.

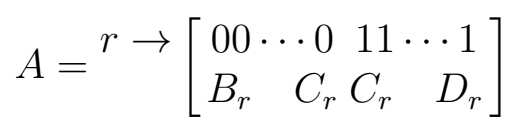

Our typical use of this is to note that $\left[B_{r} C_{r} D_{r}\right]$ is simple with no configurations $\left\{I_{2} \times I_{2}, T_{2} \times T_{2}\right\}$ and $C_{r}$ is simple with no configurations $F_{4}, F_{5}$ :

$$
F_{4}=\left[\begin{array}{ll}
1 & 1 \\
1 & 1 \\
0 & 1
\end{array}\right], \quad F_{5}=\left[\begin{array}{llll}
1 & 1 & 0 & 0 \\
1 & 0 & 1 & 0 \\
0 & 1 & 0 & 1
\end{array}\right]
$$

In particular if $C_{r}$ has $F_{4}$, then $A$ has $T_{2} \times T_{2}$, and if $C_{r}$ has $F_{5}$, then $A$ has $I_{2} \times I_{2}$, both forbidden configurations. We deduce by induction (on $m$ ) that $\|A\|=\left\|\left[B_{r} C_{r} D_{r}\right]\right\|+$ 
$\left\|C_{r}\right\| \leq \operatorname{forb}\left(m-1,\left\{I_{2} \times I_{2}, T_{2} \times T_{2}\right\}\right)+$ forb $\left(m-1,\left\{F_{4}, F_{5}\right\}\right)$. We would be done if we could show that $\left\|C_{r}\right\| \leq 20 \mathrm{~m}^{1 / 2}$ for some $r$. The choice of 20 is an artifact of our proof. We may now assume $\left\|C_{r}\right\| \geq 20 \mathrm{~m}^{1 / 2}$ for all $r$ and will arrive at a contradiction. Our proof will show that for each $r \in[m]$, we can choose a set of rows $S(r) \subseteq[m] \backslash r$ where $|S(r)| \geq\left\|C_{r}\right\| / 4 \geq 5 m^{1 / 2}$. Note that $S(r)$ is a set so $|S(r)|$ denotes cardinality while $\left\|C_{r}\right\|$ denotes the number of columns. Choose one row $r$. We show that there is a subset $K \subseteq S(r)$, of size $|S(r)| / 3$, every pair of rows $r_{i}, r_{j} \in K$ satisfying

$$
\left|S\left(r_{i}\right) \cap S\left(r_{j}\right)\right| \leq 5 .
$$

Then if we let $t=m^{1 / 2}$ we can choose $r_{1}, r_{2}, \ldots, r_{t} \in K \subseteq S(r)$ and obtain $t$ disjoint sets

$$
S\left(r_{1}\right), S\left(r_{2}\right) \backslash S\left(r_{1}\right), S\left(r_{3}\right) \backslash\left(S\left(r_{1}\right) \cup S\left(r_{2}\right)\right), \ldots, S\left(r_{t}\right) \backslash\left(S\left(r_{1}\right) \cup S\left(r_{2}\right) \cup \cdots \cup S\left(r_{t-1}\right)\right) .
$$

This yields that $S\left(r_{1}\right) \cup S\left(r_{2}\right) \cup S\left(r_{3}\right) \cdots \cup S\left(r_{t}\right)$ is of size at least

$$
5 m^{1 / 2}+\left(5 m^{1 / 2}-5\right)+\left(5 m^{1 / 2}-10\right)+\cdots>m,
$$

a contradiction given that we have $m$ rows.

Consider the following operation on $C_{r}$. Delete as many rows as we can while preserving simplicity of the remaining matrix. Doing this may involve some choices. The remaining set of rows is denoted $R(r)$ and so $\left.C_{r}\right|_{R(r)}$ is simple and $\left\|C_{r}\right\|=\left\|\left.C_{r}\right|_{R(r)}\right\|$. Now by Lemma 8 found below, $\left\|\left.C_{r}\right|_{R(r)}\right\| \leq 2|R(r)|$ and so $|R(r)| \geq\left\|C_{r}\right\| / 2$.

We consider the standard decomposition applied to $\left.C_{r}\right|_{R(r)}$

$$
\left.C_{r}\right|_{R(r)}=s \rightarrow\left[\begin{array}{cccc}
00 & \cdots & 11 & \cdots \\
E_{s} & G_{s} & G_{s} & H_{s}
\end{array}\right]
$$

Given our choice for $R(r)$, we deduce that $1 \leq\left\|G_{s}\right\|$. As in the proof of Lemma 8, we note that $G_{s}$ does not have the configurations $\left[\begin{array}{l}1 \\ 1\end{array}\right], I_{2}$. We deduce that $1 \leq\left\|G_{s}\right\| \leq 2$ where $G_{s}$ will either consist of a column of 0's or a column of sum 1 or both. If $G_{s}$ has a column of 0's then $\left.C_{r}\right|_{R(r)}$ has a column of sum 1 with a 1 in row $s$. If $G_{s}$ has a column of sum 1 with a 1 in row $t$, then $\left.C_{r}\right|_{R(r)}$ has a column of sum 2 with 1's in rows $s, t$ and also a column of sum 1 with a 1 in row $t$. In this latter case record a directed arc $s \rightarrow t$ and in this way form a directed graph $D$ on the rows $R(r)$ with at most $|R(r)| \operatorname{arcs}$. We now indicate how to find a set $S(r) \subseteq R(r)$ so that $\left.C_{r}\right|_{S(r)}$ has $I_{|S(r)|}$ and $|S(r)| \geq|R(r)| / 2$. First let $T$ denote the set of rows $t \in R(r)$ for which $\left.C_{r}\right|_{R(r)}$ has a column of sum 1 with a 1 in row $t$. Let $U=R(r) \backslash T$. Then for each $u \in U$, there is exactly one arc $u \rightarrow v$ in $D$ and, by our choice of $T, v \in T$. Let $V=\{v \in T:$ there is a $u \in U$ with $u \rightarrow v\}$. We may now form $S(r)=U \cup(T \backslash V)$. We see that $\left.C_{r}\right|_{S(r)}$ has $I_{|S(r)|}$ since for each $t \in T \backslash V$, we take the column of sum 1 with a single 1 in row $t$ and for $u \in U$, we take the column with sum 2 with 1's in rows $u, v$. In this latter case we have $u \rightarrow v$ and so $v \in V$ hence $v \notin S(r)$. We may verify $|S(r)| \geq|R(r)| / 2$ by noting that $|S(r)|=|R(r)|-|V|$ and $|V| \leq|U|$.

We need more detailed information and begin by computing what happens on quadruples of rows $\{i, j, k, \ell\}$ of $A$ in order to avoid the two $4 \times 4$ configurations $I_{2} \times I_{2}$ and $T_{2} \times T_{2}$. There are 5 cases $Q_{0}, Q_{1}, \ldots, Q_{4}$. These cases were computed using a C++ program (can be downloaded at[2]) that had many test runs checking correctness and was also independently checked by a program written in sage (public code that uses Python). In each case one may easily check that, if the case is satisfied, indeed our three matrices 
of (1) aren't present as configurations. But checking completeness of the list without a computer would require an enormous amount of work. In general we would write

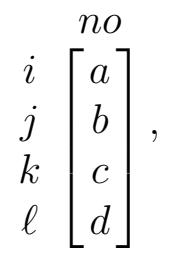

to denote that in $A$ we do not have the specified vector $(a, b, c, d)^{T}$ on rows $\{i, j, k, \ell\}$ in that order. In what follows, the row order is not specified but is the same for each column for a given case $Q_{i}$. In each case, either 3 or 4 or 5 or 6 columns must not be present on a quadruple of rows in order to not contain the configurations $\left\{I_{2} \times I_{2}, T_{2} \times T_{2}\right\}$.

$$
\begin{gathered}
n o=n o \\
Q_{0}=\left[\begin{array}{l}
0 \\
1 \\
0 \\
1
\end{array}\right]\left[\begin{array}{l}
0 \\
0 \\
1 \\
1
\end{array}\right]\left[\begin{array}{l}
1 \\
1 \\
1 \\
1
\end{array}\right], \quad Q_{1}=\left[\begin{array}{l}
0 \\
1 \\
0 \\
1
\end{array}\right]\left[\begin{array}{l}
1 \\
1 \\
0 \\
1
\end{array}\right]\left[\begin{array}{l}
0 \\
0 \\
1 \\
1
\end{array}\right]\left[\begin{array}{l}
1 \\
0 \\
1 \\
1
\end{array}\right]\left[\begin{array}{l}
0 \\
1 \\
1 \\
1
\end{array}\right], \quad Q_{2}=\left[\begin{array}{l}
1 \\
1 \\
1 \\
0
\end{array}\right]\left[\begin{array}{l}
0 \\
1 \\
0 \\
1
\end{array}\right]\left[\begin{array}{l}
0 \\
0 \\
1 \\
1
\end{array}\right]\left[\begin{array}{l}
1 \\
0 \\
1 \\
1
\end{array}\right], \\
Q_{3}=\left[\begin{array}{l}
1 \\
1 \\
1 \\
0
\end{array}\right]\left[\begin{array}{l}
1 \\
0 \\
0 \\
1
\end{array}\right]\left[\begin{array}{l}
0 \\
1 \\
0 \\
1
\end{array}\right]\left[\begin{array}{l}
0 \\
0 \\
1 \\
1
\end{array}\right], \quad Q_{4}=\left[\begin{array}{l}
1 \\
1 \\
0 \\
0
\end{array}\right]\left[\begin{array}{l}
1 \\
0 \\
1 \\
0
\end{array}\right]\left[\begin{array}{l}
0 \\
1 \\
1 \\
0
\end{array}\right]\left[\begin{array}{l}
1 \\
0 \\
0 \\
1
\end{array}\right]\left[\begin{array}{l}
0 \\
1 \\
0 \\
1
\end{array}\right]\left[\begin{array}{l}
0 \\
0 \\
1 \\
1
\end{array}\right] .
\end{gathered}
$$

In what follows we analyze closely each of the 5 cases above to deduce that the columns of $\left.\left[B_{r} C_{r}\right]\right|_{S(r)}$ form a laminar family in order to help prove (6).

If we are missing $(a, b, c, d)^{T}$ on the quadruple of rows $r, i, j, k$, then we are missing $(b, c, d)^{T}$ on the triple of rows $i, j, k$ in $C_{r}$ else $A$ has both $(0, b, c, d)^{T}$ and $(1, b, c, d)^{T}$ on rows $r, i, j, k$, a contradiction. Thus it is possible to determine what is missing in $\left.C_{r}\right|_{R(r)}$ on the triple of rows $i, j, k \in R(r)$ by considering what is missing on the quadruple of rows $r, i, j, k$. We also obtain a contradiction, based on the choice of $R(r)$, if we find a copy of ' $K_{2}$ ' in what is missing, namely if on the triple $i, j, k$ there is a pair of rows $i, j$ with all 4 columns of $K_{2}$ appearing as follows:

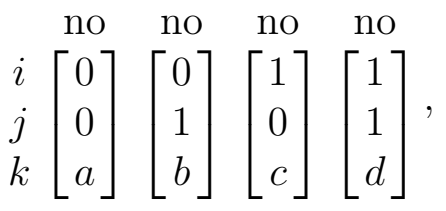

where $a, b, c, d \in\{0,1\}$. Perhaps other columns are missing on rows $i, j, k$. Note that we could delete row $k$ from $\left.C_{r}\right|_{R(r)}$ and preserve simplicity, contradicting our choice of $R(r)$. The reason for this is that on the three rows $i, j, k$, the columns present would possibly be

$$
\begin{aligned}
& i \\
& j \\
& k
\end{aligned}\left[\begin{array}{l}
0 \\
\bar{a}
\end{array}\right] \quad\left[\begin{array}{l}
0 \\
1 \\
\bar{b}
\end{array}\right] \quad\left[\begin{array}{l}
1 \\
0 \\
\bar{c}
\end{array}\right] \quad\left[\begin{array}{l}
1 \\
1 \\
\bar{d}
\end{array}\right],
$$

where $\bar{x}$ denotes the $(0,1)$-complement of $x$. We can see that deleting row $k$ will not result in repeated columns assuming $\left.C_{r}\right|_{R(r)}$ has no repeated columns. 
Consider the quadruple of rows $r, i, j, k$. We note that $Q_{3}, Q_{4}$ each contain 3 rows (rows 1,2,3 in each case), each pair of rows of which 'has a $K_{2}$ ' in what is missing and so any choice for $r$, will leave that on the remaining triple of rows in $C_{r}$ 'has a $K_{2}$ '. For $\{i, j, k\} \subseteq R(r)$, the quadruple of rows $r, i, j, k$ cannot be in cases $Q_{3}$ or $Q_{4}$. Case $Q_{0}$ 'has no $K_{2}$ ' and so if $Q_{0}$ applies to a quadruple of rows, then apparently any row of $Q_{0}$ as given could be row $r$. Case $Q_{1}$ has 'has a $K_{2}$ ' on rows 1,2 and also on rows 1,3 and so row $r$ would have to be row 1 of $Q_{1}$ as given. Case $Q_{2}$ 'has a $K_{2}$ ' on rows 1,2 and so row $r$ must either be row 1 or row 2 of $Q_{2}$ as given. This produces the following cases. We use $P_{i}$ to denote a triple arising from the quadruple $Q_{i}$ in these ways.

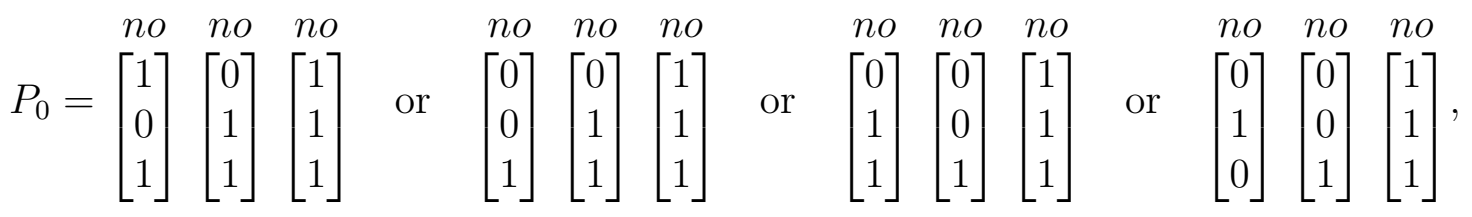

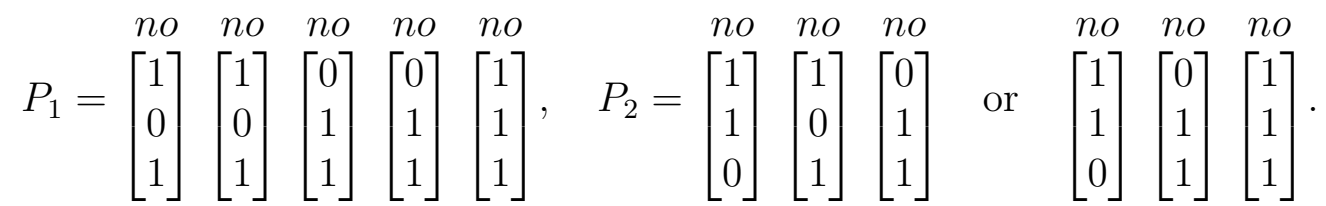

These then, are the only cases we need consider for what is missing on a triple of rows of $R(r)$ in $C_{r}$. Because we know that the columns of $\left.C_{r}\right|_{S(r)}$ contain an identity matrix $I_{|S(r)|}$ on the rows $S(r)$, we know that the final three cases of $P_{0}$ and the second case of $P_{2}$ cannot be what is missing on a triple of rows in $S(r)$. Thus $r$ would have to be the first row in $Q_{0}, Q_{1}$ or $Q_{2}$ as given.

Among the remaining options for what is missing $\left(P_{1}\right.$, the first case for $P_{2}$ and the first case for $P_{0}$ ), by looking at $Q_{0}, Q_{1}$ and $Q_{2}$, we see that below the zeroes in row $r$, all triple of rows in $S(r)$ are missing two columns of sum 2. This means that the columns in $\left.\left[B_{r} C_{r}\right]\right|_{S(r)}$ must form a laminar family. We will use this fact in what follows.

Let $r_{j} \in S(r)$. The columns of $C_{r_{j}}$ must correspond to columns of $A$ which appear with a 1 and 0 in row $r_{j}$ and are the same elsewhere, in particular, on the rows $(r \cup S(r)) \backslash r_{j}$. We thus have pairs of columns in $\left.A\right|_{S(r) \cup r}$ from $C_{r_{j}}$ as follows:

$$
\underset{r}{S(r) \backslash r_{j}\{}\left[\begin{array}{ll}
0 & 1 \\
\alpha & \alpha \\
a & a
\end{array}\right] .
$$

We begin with the case where $a=0$ by considering $\left.\left[B_{r} C_{r}\right]\right|_{S(r)}$. Suppose there exist two non-zero choices for $\alpha$, say $\beta \neq \gamma$. We have the following situation for columns in $\left.A\right|_{S(r) \cup r}$ from $C_{r_{j}}$ :

$$
S(r) \backslash r_{j}\left\{\begin{array}{llll}
r_{j} & {\left[\begin{array}{llll}
0 & 1 & 0 & 1 \\
\beta & \beta & \gamma & \gamma \\
0 & 0 & 0 & 0
\end{array}\right] .}
\end{array}\right.
$$

We know that the columns of $\left.\left[B_{r} C_{r}\right]\right|_{S(r)}$ form a laminar family. Thus the columns from (9) must form a laminar family. Columns 2 and 4 have 1's in common on row $r_{j}$ and so the columns as sets are not disjoint so one must be contained in the other. We deduce 
without loss of generality that $\beta \leq \gamma$. Now, considering columns 2,3 , and the fact that $\beta \neq \mathbf{0}$, we violate the laminar property, a contradiction. Thus there is only one non-zero choice for $\alpha$ in (8) when $a=0$ but since $\left.C_{r_{j}}\right|_{S(r)}$ need not be simple, the column with $\alpha$ could be repeated. If $\alpha$ has column sum at least 2 with 1's on rows $a, b \in S(r)$, and it is repeated, it creates a $T_{2} \times T_{2}$ as follows. This happens because $A$ is a simple matrix and so on some row $r^{\prime}\left(r^{\prime} \notin S(r)\right)$ the columns must differ, yielding the following, with $T_{2} \times T_{2}$ on rows $r_{j}, a, b, r^{\prime}$.

$$
\begin{array}{r}
r_{j} \\
a \\
b \\
b\} \\
r^{\prime}
\end{array}\left[\begin{array}{cccc}
0 & 0 & 1 & 1 \\
1 & 1 & 1 & 1 \\
1 & 1 & 1 & 1 \\
\alpha^{\prime} & \alpha^{\prime} & \alpha^{\prime} & \alpha^{\prime} \\
0 & 1 & 0 & 1
\end{array}\right]
$$

Thus if any $\alpha$ is repeated, it must have column sum 1 . We now consider the second case when $a=1$ in (8). This case will be simpler since columns with $a=1$ will automatically yield a row of ones on row $r$, and $T_{2} \times T_{2}$ has a row of 1's. Suppose there are two non-zero columns $\beta, \gamma$ in $\left.C_{r_{j}}\right|_{S(r) \backslash r_{j}}$. Since $\left.C_{r_{j}}\right|_{S(r) \backslash r_{j}}$ need not be simple, we do not require $\beta \neq \gamma$. In some columns of $\left.A\right|_{S(r) \cup r}$ from $C_{r_{j}}$ we have

$$
S(r) \backslash r_{j}\left\{\begin{array}{llll}
r_{j} \\
r
\end{array}\left[\begin{array}{llll}
0 & 0 & 1 & 1 \\
\beta & \gamma & \beta & \gamma \\
1 & 1 & 1 & 1
\end{array}\right] .\right.
$$

Now suppose $\beta$ and $\gamma$ both have entry 1 on some row $r^{\prime} \in S(r) \backslash r_{j}$. Also, as above, because $A$ is a simple matrix, there is some row $r^{\prime \prime}\left(r^{\prime \prime}\right.$ need not be in $\left.S(r)\right)$ where the columns differ. The situation is then as follows:

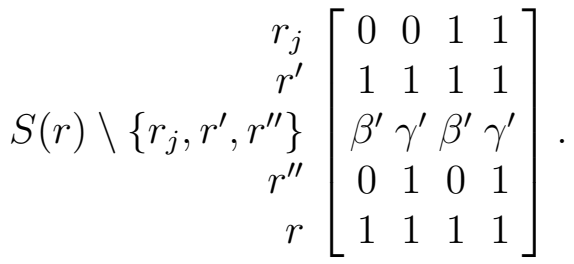

This creates $T_{2} \times T_{2}$ in $A$. Therefore for any pair of non-zero columns $\alpha$ in $\left.C_{r_{j}}\right|_{S(r) \backslash r_{j}}$, with 1 's in row $r$, there cannot be a row in $S(r) \backslash r_{j}$ where both are 1's.

We have shown that for the columns in $\left.C_{r_{p}}\right|_{S(r) \cup\{r\}}$ which are 0 in row $r$, then those columns can be $\mathbf{0}$ and/or a single non-zero column $\alpha$. If $\alpha$ is of column sum 1 it may be repeated, otherwise there is only one copy in $\left.C_{r_{p}}\right|_{S(r) \cup\{r\}}$. We have also shown that for the columns in $\left.C_{r_{p}}\right|_{S(r) \cup\{r\}}$ which are 1 in row $r$, that there is no row in $S(r) \backslash r_{p}$ where more than one of the columns have 1's.

Let $r_{p} \in S(r)$ be given. We say that row $r_{s} \in S(r) \backslash r_{p}$ is a bad row for $r_{p}$ if more than two columns in $\left.C_{r_{p}}\right|_{S(r)}$ have 1's in row $r_{s}$. By our case analysis above, we see that there is at most one bad row for a given row $r_{p}$ which will arise from columns which are 0's in row $r$ and have a column $\alpha$ of sum 1 repeated plus possibly with a column which is 1 in row $r$. For all rows $r_{q} \in S(r) \backslash r_{p}$, except possibly a single bad row, there is at most one column of the columns of $C_{r_{p}}$ with a 0 in row $r$, which also has a 1 in row $r_{q}$ and there is at most one column of the columns of $C_{r_{p}}$ with a 1 in row $r$, which also has a 1 in row $r_{q}$. Thus for all rows in $S(r) \backslash r_{p}$ with the exception of the previously described bad row, the remaining rows will have at most two 1's on the columns of $\left.C_{r_{p}}\right|_{S(r) \backslash r_{p}}$. 
Now given a pair $r_{p}, r_{q}$, for which neither is a bad row for the other, we can now show that $\left|S\left(r_{p}\right) \cap S\left(r_{q}\right)\right| \leq 5$. Assume the contrary, that $\left|S\left(r_{p}\right) \cap S\left(r_{q}\right)\right| \geq 6$. We decompose $A$ first using $r_{p}$ and then using $r_{q}$. In each case we only show those column of $A$ arising from $C_{r_{p}}$ (respectively from $C_{r_{q}}$ ) which yield the identity matrix on the rows $S\left(r_{p}\right) \cap S\left(r_{q}\right)$. Let $t=\left|S\left(r_{p}\right) \cap S\left(r_{q}\right)\right| \geq 6$.

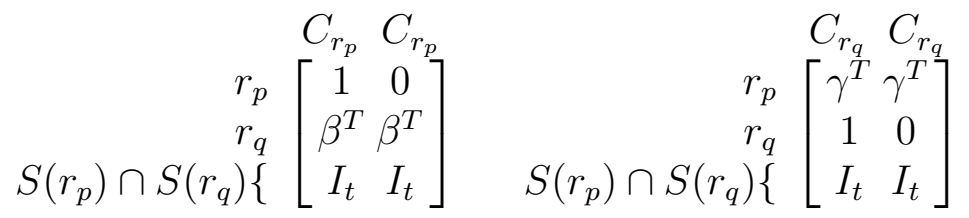

Let $\beta=\left(b_{1}, b_{2}, \ldots, b_{t}\right)^{T}$ and $\gamma=\left(g_{1}, g_{2}, \ldots, g_{t}\right)^{T}$ and let $J=\left\{j: b_{j}=g_{j}=0\right\}$. By assumption, $\beta^{T}$ and $\gamma^{T}$ each can contain at most two 1's and so $|J| \geq t-4$. We now consider the columns indexed by $J$ and the rows indexed by $\{1,2\} \cup J^{\prime}$ where $J^{\prime}=\{j+2$ : $j \in J\}$ for each of the following $(t+2) \times t$ matrices:

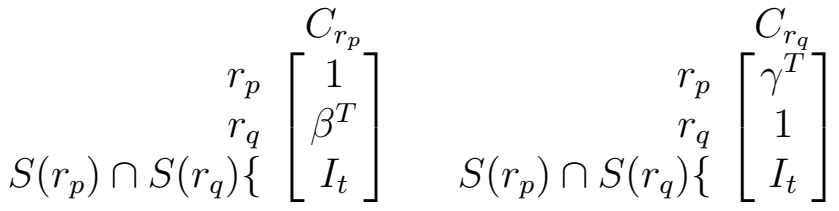

This yields $I_{2} \times I_{t-4}$ and hence $I_{2} \times I_{2}$ in $A$, a contradiction. Therefore $\left|S\left(r_{p}\right) \cap S\left(r_{q}\right)\right| \leq 5$.

It remains to show that we can choose sufficient rows from $S(r)$ such that each row $r_{q}$ is not a bad row for all other rows chosen. However, this is straightforward when interpreted as a graph theory problem. We create a graph with $|S(r)|$ vertices and assign each row of $S(r)$ to a vertex. We assign an arc $r_{i} \rightarrow r_{j}$ if $r_{j}$ is a bad row for $r_{i}$. Because for each row $r_{i}$ there can only be one bad row $r_{j}$, the out-degree of any vertex $r_{i}$ is at most one. A set of rows that has our desired property is equivalent to choosing an independent set in our graph. By Lemma 9 we know that it is possible to choose such a set that is at least a third of the remaining rows. Thus we can choose at least $|S(r)| / 3$ rows where for any chosen rows $r_{p}, r_{q}$, neither row is a bad row for the other and so $\left|S\left(r_{p}\right) \cap S\left(r_{q}\right)\right|<6$. We then generate the series of disjoint sets described at the beginning of this proof and arrive at our contradiction.

Lemma 8. We have that forb $\left(m,\left\{F_{4}, F_{5}\right\}\right) \leq 2 m$.

Proof: Let $A$ be an $m$-rowed simple matrix with no configurations $F_{4}, F_{5}$. We apply the standard decomposition of (4) and note that $C_{r}$ does not contain configurations $\left[\begin{array}{l}1 \\ 1\end{array}\right]$ or $I_{2}$ else $A$ has $F_{4}$ and $F_{5}$ respectively. But then $\left\|C_{r}\right\| \leq 2$ and can either have no columns or a column of 0's or a column of sum 1 or two columns consisting of a column of 0's and a column of sum 1 . Then by induction, $\|A\|=\left\|\left[B_{r} C_{r} D_{r}\right]\right\|+\left\|C_{r}\right\| \leq \operatorname{forb}\left(m-1,\left\{F_{4}, F_{4}\right\}\right)+2$, which yields the bound.

Lemma 9. Given a directed graph $D=(V, A)$ where for all $v \in V$ the maximum outdegree is 1 , it is always possible to colour the vertices of $D$ with 3 colours so that if $u \rightarrow v \in A$ then $u$ and $v$ have different colours.

Proof: We use induction on the number of vertices. Suppose there exists a $v^{\prime} \in V$ such that $v^{\prime}$ has in-degree 0 . Let $D^{\prime}$ be the induced subgraph of $D \backslash v^{\prime}$. Directed graph $D^{\prime}$ maintains our property of maximum out-degree 1 , so we colour $D^{\prime}$ with 3 colors and then 
add $v^{\prime}$ back with an appropriate colour. Suppose there is no vertex $v \in V$ with in-degree 0 . In this case all vertices have in-degree 1 and out-degree 1 , and so the edges of $D$ form the union of disjoint cycles, which we can colour with 3 colours.

It is likely (but unknown) that forb $\left(m,\left\{E_{1}, E_{2}, E_{3}\right\} \times\left\{E_{1}, E_{2}, E_{3}\right\}\right)$ is $O\left(m^{3 / 2}\right)$. One might ask the relationship of Theorem 4 to Conjecture 1. The Conjecture (which applies only for a single forbidden configuration) says that only product constructions are needed for best possible asymptotics, but in this case $\left\{I_{2} \times I_{2}, T_{2} \times T_{2}\right\}$ are simultaneously missing from all 1-fold products. In particular $I \times I$ avoids $T_{2} \times T_{2}$ but does not avoid $I_{2} \times I_{2}$ (Proposition 1). Surprisingly there is an $O\left(\mathrm{~m}^{3 / 2}\right)$ construction contained in $I \times I$ and yet avoiding $I_{2} \times I_{2}$ (Theorem 1). The other 2-fold products $I_{2} \times T_{2}$ (Theorem 2) and $T_{2} \times T_{2}$ (Theorem 3) behave as the conjecture might suggest.

\section{A bound for $104 \times 4$ Forbidden Configurations}

Proof of Theorem 5: Use the notation $\mathcal{F}=\left\{I_{2}, T_{2}, U_{2}, V_{2}\right\} \times\left\{I_{2}, T_{2}, U_{2}, V_{2}\right\}$. We establish the lower bound by construction. Let $\alpha=\mathbf{1}_{m-1} \mathbf{0}_{1}$. We construct the $m \times(m+3)$ matrix $A$ consisting of $\left[\mathbf{0}_{m} I \alpha \mathbf{1}_{m}\right]$. Not that each of $\left\{I_{2}, T_{2}, U_{2}\right\}$ has one column of sum 1 and hence each matrix $\left\{I_{2}, T_{2}, U_{2}\right\} \times\left\{I_{2}, T_{2}, U_{2}\right\}$ has a column of sum 2 but no column of $A$ thas at least 21 's and 20 's. The same observation holds for $T_{2} \times V_{2}, U_{2} \times V_{2}$, and $V_{2} \times V_{2}$. Now $I_{2} \times V_{2}$ has its first two rows containing $\left[I_{2} \mid I_{2}\right]$ yet no two rows of $A$ have $\left[I_{2} \mid I_{2}\right]$ and so $A$ has no $I_{2} \times V_{2}$. Hence $A$ avoids all configurations in $\mathcal{F}$, thus forb $(m, \mathcal{F}) \geq m+3$.

We use induction on $m$ for the upper bound. Begin by verifying forb $(4, \mathcal{F})=7$ using a $\mathrm{C}++$ program which exhaustively considered all subsets of 8 columns of $K_{4}$ and verified that in all of them $F$ is contained as a configuration. To prove the bound for $m \geq 5$, we will proceed by induction on $m$. For an $m$-rowed matrix $A$ that doesn't contain any configuration in $\mathcal{F}$ it suffices by induction to show there exists a row $r$ for which $\left\|C_{r}\right\| \leq$ 1 , using the standard decomposition as in (7). If this were so, we could delete row $r$ and perhaps one column (one instance of the column forming $C_{r}$ ) from $A$, keeping the remaining matrix simple. This would yield forb $(m, \mathcal{F}) \leq 1+\operatorname{forb}(m-1, \mathcal{F})=1+(m-$ 1) $+3=m+3$ as desired.

Let us proceed by contradiction. Suppose then that for every row $r,\left|C_{r}(A)\right| \geq 2$. We then have at least two columns $\alpha$ and $\beta$ in $C_{1}(A)$. The matrix $A$ would look like this

$$
1\left[\begin{array}{cccccccc}
0 & \cdots & 0 & 0 & 1 & 1 & \cdots & 1 \\
& \alpha & \beta & \alpha & \beta & & &
\end{array}\right] .
$$

But $\alpha$ and $\beta$ must differ in some row. Without loss of generality, assume they differ on row 2, and suppose $\alpha_{2}=0$ and $\beta_{2}=1$. We will prove that $\alpha$ and $\beta$ must be $(0,1)$-complements of each other. Suppose otherwise and suppose they had something in common, say in row 3 . The first four rows of $A$ would look like this (where we are not requiring $b \neq c)$ :

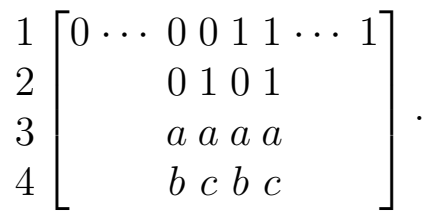


for some values of $a, b, c$ (we are using the fact that the matrix has at least 4 rows). Then in rows 1 and 3 and rows 2 and 4 we get that this matrix contains

$$
\begin{aligned}
& \begin{array}{c}
1 \\
3\left[\begin{array}{ll}
0 & 1 \\
a & a
\end{array}\right] \\
\times
\end{array} . \\
& \begin{array}{l}
2 \\
4
\end{array}\left[\begin{array}{ll}
0 & 1 \\
b & c
\end{array}\right]
\end{aligned}
$$

which is a configuration of $\mathcal{F}$ (for any $a, b, c$ ), so we conclude $\alpha=\bar{\beta}$.

Now $C_{2}(A)$ must have two repeated columns, say $\gamma$ and $\delta$. As argued above, they must be $(0,1)$-complements. Here is part of the matrix $A$ :

$$
\left[\begin{array}{cccccccc}
0 & 0 & 0 & 0 & 1 & 1 & 1 & 1 \\
0 & 0 & 1 & 1 & 0 & 0 & 1 & 1 \\
\alpha & \gamma & \alpha & \bar{\gamma} & \bar{\alpha} & \gamma & \bar{\alpha} & \bar{\gamma}
\end{array}\right] .
$$

Since $\alpha$ and $\bar{\gamma}$ have to differ somewhere, we can assume $\alpha_{3}=a$, and $\gamma_{3}=a$. Since $\alpha$ and $\gamma$ must differ somewhere, we can assume $\alpha_{4}=b$ and $\gamma_{4}=\bar{b}$. Furthermore, since we have at least 5 rows, we can then write the selected columns of $A$ where the columns are given labels below to indicate the source of the column.

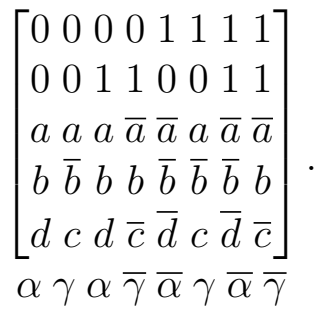

There are two cases. Either $d=c$ or $d=\bar{c}$. So we either have

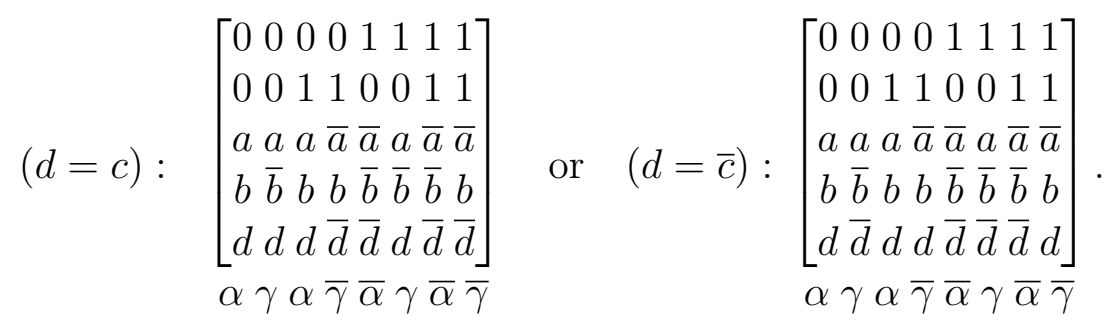

These yield the following configurations in $\mathcal{F}$ respectively:

$$
\begin{array}{cc}
2\left[\begin{array}{ll}
0 & 1 \\
\bar{b} & b
\end{array}\right] & 2\left[\begin{array}{ll}
0 & 1 \\
a & \bar{a}
\end{array}\right] \\
\times & \times \\
3\left[\begin{array}{ll}
a & \bar{a} \\
d & \bar{d}
\end{array}\right] & 4\left[\begin{array}{ll}
b & \bar{b} \\
d & \bar{d}
\end{array}\right]
\end{array} .
$$

This is a contradiction to $\left\|C_{r}\right\| \geq 2$ and hence for $m \geq 5$, there must be some row $r$ for which $\left|C_{r}(A)\right| \leq 1$ which then yields the bound by induction. 


\section{References}

1. R.P. Anstee, A Survey of Forbidden Configurations results, http://www.math.ubc.ca/ anstee.

2. R.P. Anstee, A Survey of Forbidden Configurations results, http://www.math.ubc.ca/ anstee/FCConfThesisVersion.tar.gz

3. R.P. Anstee, Balin Fleming, Two refinements of the bound of Sauer, Perles and Shelah and Vapnik and Chervonenkis, Discrete Math., to appear.

4. R.P. Anstee, J.R. Griggs, A. Sali, Small Forbidden Configurations, Graphs and Combinatorics 13(1997), 97-118.

5. R.P. Anstee, A. Sali, Small Forbidden Configurations IV, Combinatorica 25(2005), 503-518.

6. Anstee, R. P., B. Fleming, Z. Füredi, and A. Sali, Color critical hypergraphs and forbidden configurations, proceedings of EuroComb 2005, Berlin, Germany. Discrete mathematics and Theoretical Computer Science, 2005, 117-122.

7. J. Balogh, B. Bollobás, Unavoidable Traces of Set Systems, Combinatorica, 25 (2005), 633643.

8. J. Balogh, B. Bollobás, R. Morris, Hereditary properties of partitions, ordered graphs and ordered hypergraphs, Eur. J. Combin., 8 (2006), 1263-1281.

9. P. Erdős, On Extremal Problems of Graphs and Generalized Graphs, Israel J. Math., 2(1964), 183-190.

10. P. Erdős and M. Simonovits, A limit theorem in graph theory. Studia Sci. Math. Hungar 1 (1966), 51-57.

11. P. Erdős, A.H. Stone, On the Structure of Linear Graphs, Bull. A.M.S., 52(1946), 1089-1091.

12. Z. Füredi, An upper bound on Zarankiewicz problem, Combinatorics, Probability and Computing 5(1996), 29-33.

13. Z. Füredi, P. Hajnal, Davenport-Schinzel theory of matrices, Discrete Math. 103(1992), 233-251.

14. M. Klazar, A. Marcus, Extensions of the linear bound in the Füredi-Hajnal conjecture, Adv. in Appl. Math., 38 (2007), 258-266.

15. Kővari, V. Sós, P. Turán, On a problem of K. Zarankiewicz, Colloq. Math 3 (1954), 50-57.

16. A. Marcus, G. Tardos, Excluded permutation matrices and the Stanley Wilf Conjecture, J. Combin. Th. Ser. A 107 (2004), 153-160.

17. G. Tardos, On 0-1 matrices and small excluded submatrices, J. Combin. Th. Ser. A 111 (2005), 266-288. 